\title{
Pressure overload promotes cystatin C secretion of cardiomyocytes to regulate the MAPK signaling pathway and mediate cardiac hypertrophy
}

\author{
Yi Shen ${ }^{1 \#}$, Xiaoyi Zhang ${ }^{1 \#}$, Chenguang $\mathrm{Li}^{2 \#}$, Xiang Wang ${ }^{2}$, Yong Ye $^{2}$, Jie Yuan ${ }^{2}$, Hui Gong ${ }^{2}$, Yunzeng Zou ${ }^{2}$, \\ Junbo $\mathrm{Ge}^{2}$ \\ ${ }^{1}$ Department of Geriatrics, Zhongshan Hospital, Fudan University, Shanghai, China; ${ }^{2}$ Shanghai Institute of Cardiovascular Diseases, Zhongshan \\ Hospital, Fudan University, Shanghai, China \\ Contributions: (I) Conception and design: Y Zou, H Gong, Y Shen; (II) Administrative support: Y Zou, J Ge; (III) Provision of study materials or \\ patients: J Ge; (IV) Collection and assembly of data: Y Shen, C Li; (V) Data analysis and interpretation: Y Shen, J Yuan, Y Zou; (VI) Manuscript \\ writing: All authors; (VII) Final approval of manuscript: All authors. \\ \#These authors contributed equally to this work. \\ Correspondence to: Yunzeng Zou. Shanghai Institute of Cardiovascular Diseases, Zhongshan Hospital, Fudan University, Shanghai, China. \\ Email: zou.yunzeng@zs-hospital.sh.cn.
}

Background: This study aimed to compare serum cystatin C (CysC) levels between hypertensive and nonhypertensive patients, and to explore the correlation between serum $\mathrm{Cys} C$ and left ventricular hypertrophy (LVH). We also investigated the effects of pressure overload on cardiac expression and secretion of CysC, and explored the direct effect of $\mathrm{Cys} C$ on the hypertrophy of primary cardiomyocytes.

Methods: Serum CysC was compared in patients with hypertension (634 patients) and those without hypertension (411 patients), and the correlation between serum CysC levels and $\mathrm{LVH}$ was explored. A transverse aortic constriction (TAC) mouse model and a mechanical stretch model of primary cardiomyocytes and fibroblasts were developed to compare cardiac expression and secretion of $\mathrm{Cys} C$ under pressure overload. After intervention with exogenous CysC, we compared the cross-sectional area of primary cardiomyocytes, cardiac hypertrophy-associated gene expression, and phosphorylation of the MAPK signaling pathway.

Results: In chronic kidney disease (CKD) stage 1 patients, serum CysC was higher in hypertensive patients independent of renal function. Serum CysC elevation was an independent predictor of $\mathrm{LVH}$ after correction for endogenous creatinine clearance rate (eCCr), left ventricular ejection fraction (LVEF), and NT-proBNP. Cardiac levels of CysC in TAC mice were elevated. CST3 gene expression was upregulated, and both intracellular and culture supernatant $\mathrm{CysC}$ levels increased after mechanical stretch of primary cardiomyocytes. After intervention with exogenous $\mathrm{CysC}$, the cross-sectional area of primary cardiomyocytes increased, as well as the gene expression of Nppa, Nppb, and Myh7, and the phosphorylation of ERK, p38, and TAK1.

Conclusions: Serum CysC levels were higher in hypertensive patients, and serum CysC elevation was an independent predictor of $\mathrm{LVH}$ after correction for eCCr. Pressure overload induced greater cardiomyocyte secretion of CysC. Exogenous CysC can enter cardiomyocytes, having a pro-hypertrophic effect on primary cardiomyocytes through regulation of the MAPK signaling pathways.

Keywords: Cystatin C (CysC); pressure overload; cardiac hypertrophy; MAPK signaling pathway

Submitted Sep 22, 2020. Accepted for publication Nov 18, 2020.

doi: $10.21037 / \mathrm{atm}-20-7041$

View this article at: http://dx.doi.org/10.21037/atm-20-7041 


\section{Introduction}

Hypertension is a common cardiovascular disease, as well as an independent risk factor for cardiovascular and cerebrovascular disease and mortality. Primary hypertension is the result of the combined effect of multiple risk factors such as genetic factors, high sodium diet, overweight and obesity, chronic stress and lack of exercises. Most patients will require pharmacological therapy in addition to lifestyle measures to achieve optimal blood pressure control (1). In recent years, device-based therapy for hypertension became a fastmoving field, such as carotid baroreceptor stimulation (2), renal denervation (3) and creation of an arteriovenous fistula (4). Considering the long-term effectiveness and potential side effects, device-based therapies are not recommended for the routine treatment of hypertension now (1). The purpose of these therapies is to decrease target organ damage and mortality and other adverse events of patients. The incidence of hypertensioninduced target organ damage increases significantly with age and the early diagnosis and treatment of hypertension is very important.

Serum biomarkers can help to identify heart and kidney target organ damage during the early stages of hypertension. In recent years, research has focused on several new serum biomarkers. Heart-type fatty acid binding protein (H-FABP) reflects the myocyte injury. Soluble growth-stimulating expression gene 2 protein (sST2), serum growth differentiation factor-15 (GDF-15) and galectin-3 (Gal-3) are the inflammatory mediators and markers of oxidative stress, which can predict new-onset heart failure. Urine kidney injury factor-1 (KIM-1) and Urine neutrophil collagenase-associated lipocalin (NGAL) are markers of renal dysfunction and have been reported to be potential predictors of new-onset heart failure (5). Among the biomarkers cysteine protease inhibitor $\mathrm{C}$ (Cystatin C, CysC) is considered to be a serum biomarker that can simultaneously reflect hypertension-induced heart damage and renal damage. $\mathrm{Cys} C$ is a low molecular weight protein secreted by all nucleated cells of the body at a constant rate, and is particularly concentrated in body fluids. Previous studies have shown that its secretion is not affected by gender, age, fatigue, and diet, and it can be freely filtered by the glomerulus, then almost completely reabsorbed and degraded in the proximal tubule (6). Therefore, CysC has been long-regarded as a stable serum biomarker that reflects glomerular filtration function (7). Furthermore, it has been reported that elevated serum $\mathrm{Cys} C$ is also related to the risk of hypertension $(8,9)$, hypertension-induced myocardial hypertrophy (10-12), chronic and acute heart failure $(13,14)$, hypertensive nephropathy $(15,16)$, and cardiovascular disease morbidity and mortality (17-22). Some researchers have found that excluding the influence of renal function, increased serum CysC levels still have diagnostic and prognostic value $(17,18)$.

It is not clear whether $\mathrm{Cys} C$ has a direct effect on myocardial hypertrophy. In a previous study, our laboratory performed iTRAQ analysis of cultured medium from cardiomyocytes or cardiac fibroblasts treated with mechanical stretch for 24 hours compared to controls. We identified the protein secretion of $\mathrm{CysC}$ from cardiomyocytes increased under mechanical stretch. Therefore, we hypothesized that the fluctuation of serum CysC in hypertensive patients may actually reflect not only its filtration and clearance in the kidneys, but also an increase in the production and secretion of $\mathrm{Cys} C$ when the heart is under pressure overload. In the case of relatively stable circulating CysC levels, locally expressed and secreted $\mathrm{Cys} C$ in the myocardium may form a positive or negative feedback mechanism through autocrine or paracrine effects, and directly participate in the regulation of the pathogenesis of hypertension-induced myocardial hypertrophy.

Myocardial hypertrophy is the result of the combined effects of neurohumors, cytokines, and other factors. Among them, increased mechanical load is the most important cause, and MAPK signaling pathways are the most important signal pathway mediating cardiac hypertrophy (23). So we also explore the influence of CysC on MAPK signaling pathway.

We present the following article in accordance with the ARRIVE reporting checklist (available at http://dx.doi. org/10.21037/atm-20-7041).

\section{Methods}

\section{Study population}

The inclusion criteria of hypertension (HBP) group was the patients diagnosed with hypertension according to the 2013 European Society of Hypertension (ESH)/European Society of Cardiology (ESC) hypertension diagnostic criteria (24) hospitalized in the Department of Cardiology at Zhongshan Hospital of Fudan University from July 2016 to November 2016. The control group was the patients without hypertension who are hospitalized in the Department of Cardiology at the same time. The exclusion criteria

\section{9} 50 51 52 53 54 55 56 57 58 59 60 61 62 63 64 65 66 67 68 69 70 71 
were as follows: patients with secondary hypertension (including substantial renal disease, renal artery stenosis, primary hyperaldosteronism, pheochromocytoma and Cushing's syndrome), acute myocardial infarction within 1 month, renal insufficiency caused by reasons other than hypertension (including renal disease and extrarenal disease), acute heart failure, acute cerebrovascular accident, acute infection within 2 weeks, surgery or trauma, severe liver and kidney dysfunction [chronic kidney disease (CKD) stage 5], and patients unable to cooperate in the study.

All procedures performed in studies involving human participants were in accordance with the Helsinki Declaration (as revised in 2013). The study was approved by the local Ethics Committee. All participants provided written informed consent to clinical examinations, laboratory analyses, and the use of data records for research purposes.

\section{Laboratory and echocardiographic data analysis}

All the enrolled patients were asked in detail about their age, gender, history of coronary heart disease, chronic heart failure, diabetes, chronic kidney disease, and history of smoking and drinking. Blood pressure, heart rate, height and weight were measured, and Body Mass Index (BMI) and Body Surface Area (BSA) were calculated.

All parameters including serum creatinine, troponin T, NT-proBNP, glycated hemoglobin, and CysC levels of enrolled patients were measured according to standard methods in the clinical laboratory of Zhongshan Hospital of Fudan University. We evaluate the patient's renal function level with the Chronic Kidney Disease (CKD) staging criteria of The Kidney Disease: Improving Global Outcomes (KDIGO) according to the calculated endogenous creatinine clearance rate (eCCr) based on the simplified Modification of Diet in Renal Disease (MDRD) formula (25). The eCCr $>90 \mathrm{~mL} / \mathrm{min} / 1.73 \mathrm{~m}^{2}$ was CKD stage 1 means normal eCCr, the eCCr $60-89 \mathrm{~mL} / \mathrm{min} / 1.73 \mathrm{~m}^{2}$ was CKD stage 2 means mildly decreased eCCr, the eCCr $30-59 \mathrm{~mL} / \mathrm{min} / 1.73 \mathrm{~m}^{2}$ was CKD stage 3 means moderately decreased eCCr, the eCCr $15-29 \mathrm{~mL} / \mathrm{min} / 1.73 \mathrm{~m}^{2}$ was CKD stage 4 means severely decreased eCCr, and the eCCr $<15 \mathrm{~mL} / \mathrm{min} / 1.73 \mathrm{~m}^{2}$ was CKD stage 5 means kidney failure. Patients with eCCr less than $60 \mathrm{~mL} / \mathrm{min} / 1.73 \mathrm{~m}^{2}$ for $>3$ months were diagnosed with CKD.

All enrolled patients underwent resting echocardiography which measured the left atrium inner diameter (LAD), left ventricular end systolic diameter (LVESd), left ventricular 145 end diastolic diameter (LVEDd), left ventricular posterior 146 wall thickness (LVPWT), interventricular septal thickness 147 (IVST), pulmonary artery pressure (PASP), and left 148 ventricular ejection fraction (LVEF) through the apical 149 four-chamber view. Left ventricular mass (LVM) was 150 calculated using the Devereux formula (26): $\operatorname{LVM}(\mathrm{g})=0.8 \quad 151$ $\times 1.04 \times\left[(\text { LVEDd + IVST + LVPWT })^{3}-(\text { LVEDd })^{3}\right]+152$ 0.6. Left ventricular mass index (LVMi) was calculated by 153 the formula: LVMi $\left(\mathrm{g} / \mathrm{m}^{2}\right)=\mathrm{LVM} / \mathrm{BSA}$. Left atrium inner 154 diameter index (LADi) was calculated by the formula: 155 LADi $\left(\mathrm{cm} / \mathrm{m}^{2}\right)=$ LAD/BSA. The ratio of the early peak 156 left ventricular diastolic blood flow E peak to the late 157 left ventricular diastolic A peak (E/A) was measured by 158 Color Doppler flow imaging (CDFI). Patients with LVMi 159 $>125 \mathrm{~g} / \mathrm{m}^{2}$ in males and $>110 \mathrm{~g} / \mathrm{m}^{2}$ in females were 160 diagnosed with left ventricular hypertrophy (LVH) (27). 161 Patients with heart failure were diagnosed according to the 162 2016 ESC Guidelines for the diagnosis and treatment of 163 acute and chronic heart failure (28). 164

\section{Experimental animals and pressure overload mouse model}

Wild type C57BL/6 male mice aged 8-10 weeks and weighted $22-25 \mathrm{~g}$ were obtained from Shanghai Laboratory Animal Center (Chinese Academy of Sciences, Shanghai, China). A pressure overload mouse model was induced by transverse aortic constriction (TAC) as described previously (29). A total of 40 mice were randomly divided into five groups, $3,7,14$, and 28 days after TAC and sham operation, eight in each group. They were anesthetized and artificially ventilated, then the transverse aorta was ligated using a 7-0 nylon suture together with a blunted 27 -gauge needle, which was later pulled out. The sham group underwent the same surgical procedures except ligation of the transverse aorta. All animal experiments were approved by the Animal Care and Use Committee of Fudan University and in compliance with the Guidelines for the Care and Use of Laboratory Animals published by the National Academies Press (NIH publication number: 85-23, revised 1996).

The hemodynamic parameters were measured, and echocardiography was performed at different time points $(3,7,14$, and 28 days) after TAC or sham operation. Hemodynamic parameters were measured using a $1.4 \mathrm{~F}$ cardiac catheter (Millar Instruments, Inc.) connected to a Power Laboratory system (AD Instruments, Castle Hill, Australia). The catheter was inserted into the right 
common carotid artery and finally introduced into the left ventricle $(\mathrm{LV})$ to measure blood pressure (BP), LV endsystolic pressure (LVESP), and LV end-diastolic pressure (LVEDP). Echocardiography was performed using an animal-specific instrument (Visual Sonics Vevo770, Visual Sonics Inc.) as previously described (30). Briefly, mice were anesthetized with isoflurane (0.5-4\%), and the LV M-mode images were recorded. All measurements were averaged over 5 consecutive cardiac cycles. The heart tissue was then obtained for further analysis.

\section{Neonatal rat cardiomyocyte and cardiac fibroblast cell culture}

The cardiac cardiomyocytes and fibroblasts were obtained from 1-2 day old Sprague-Dawley (SD) rats using the trypsin digestion method for primary culture as described in our previous study (31). Using ophthalmic scissors, a $3 \mathrm{~cm}$ incision was made in the left rib near the sternum. After gently squeezing to expose the heart, it was cut off and placed in ice-cold PBS and washed twice. The heart was then minced into pieces and subjected to $0.1 \%$ trypsin digestion in Hank's balanced salt solution. The cell suspension was collected and placed in a Petri dish containing F12/DMEM medium with $10 \% \mathrm{FBS}$ and $1 \%$ antibiotics, then put into a cell incubator $\left(37{ }^{\circ} \mathrm{C}, 5 \% \mathrm{CO}_{2}\right)$ for 1.5 hours. Adherence of the myocardial fibroblasts, not the myocardial cells, was confirmed under a microscope. The supernatant cardiomyocytes were collected and cultured with F12/DMEM containing 10\% FBS and 1\% antibiotics for 24 hours, then the culture medium was changed every 2 days. The adherent cardiac fibroblasts were cultured in F12/DMEM containing 10\% FBS and 1\% antibiotics and passaged every 2 days.

The neonatal cardiomyocytes and cardiac fibroblasts were subjected to mechanical stretch or treated with angiotensin II (AngII, Sigma, USA) $10^{-6} \mathrm{M}$ after incubation with serum-free medium for 12 hours to perform further analyses. The neonatal cardiomyocytes were treated with different concentrations of CysC purified protein (Enzo Life Science, USA, \#BML-SE479-0100), or transfected with CysC-siRNA after incubation with serum-free medium for 12 hours to perform further analyses.

\section{Mechanical stretch in vitro}

We used a silicone sheet $(20 \mathrm{~mm} \times 40 \mathrm{~mm})$ coated with rat tail collagen in $0.1 \%$ acetic acid as the stretch device, which has been described in our previous studies $(32,33) .241$ Cardiomyocytes or cardiac fibroblasts were cultured on 242 the silicone sheets for 2-3 days, then deprived of serum for 243 12 hours. The silicone sheet was fixed in the stretching 244 frame which was put in a $150 \mathrm{~mm}$ culture dish. Uniaxial 245 strain was induced by stretching the silicone sheet in the 246 frame, and the silicon sheet was stretched to $120 \%$. The 247 control cells were also grown on the silicone sheet without 248 stretching. The cells or the culture medium were harvested 249 for analysis at specific time points ( 15 minutes, 3 hours, 250 6 hours, 12 hours and 24 hours).

\section{CysC-siRNA transfection}

We used CST3-siRNA (Silencer ${ }^{\circledR}$ Select Predesigned siRNA, Life technologies, USA) to lower the expression of Cys $\mathrm{C}$ in cardiomyocytes in vitro. The CST3-siRNA sequence was: sense (sequence $5^{\prime} \rightarrow 3^{\prime}$ ) ACAUGUACCAAGUCCCAGAtt; antisense (sequence $\left.5^{\prime} \rightarrow 3^{\prime}\right)$ UCUGGGACUUGGUACAUGUag. We used Silencer ${ }^{\circledR}$ Select GAPDH Positive Control siRNA\#1 (Life technologies, USA) as the positive control siRNA, and Silencer ${ }^{\circledR}$ Select Negative Control siRNA (Life technologies, USA) as the negative control. Lipofectamine ${ }^{\circledR}$ RNAiMAX Transfection Agent (Life technologies, USA) was used for the transfection of CST3-siRNA according to the manufacturer's instructions. Briefly, the diluted CST3-siRNA (dilute $3 \mu \mathrm{L}$ CST3-siRNA in $150 \mu \mathrm{L}$ optiDMEM) and the diluted transfection agent (dilute $9 \mu \mathrm{L}$ Lipofectamine ${ }^{\circledR}$ RNAiMAX Transfection Agent in $150 \mu \mathrm{L}$ opti-DMEM) were mixed and kept still for 5 minutes at room temperature, then the mixtures $(250 \mu \mathrm{L})$ were added to the cultured cardiomyocytes. The total RNA of the cells was extracted at 24 and 48 hours to evaluate the knockdown efficiency of the target gene, and the total protein of the cells and in the supernatant of the medium were extracted for analysis of CysC protein levels.

\section{Immunofluorescence staining}

We use anti- $\alpha$-actinin antibody immunofluorescence to visualize cardiomyocytes and calculate the crosssectional area (CSA) of cardiomyocytes. Cells were fixed in paraformaldehyde and rinsed twice with PBS after fixation. Cells were then permeabilized in immunostaining permeabilization buffer (Beyotime, Shanghai, China, \#P0097) for 10 minutes, and blocked in immunostaining blocking buffer (Beyotime, Shanghai, China, \#P0102) 
for 30 minutes. Cells were treated with anti- $\alpha$-actinin antibody (Bioss, Beijing, China, \#BS-10367R) diluted in the blocking buffer overnight. After washing 3 times in PBS for 5 minutes each, $\mathrm{Cy} 3$ labeled secondary antibody (Beyotime, Shanghai, China, \#P0183) was added to cells in blocking buffer for 1 hour. Cells were then washed 3 times with PBS, Hoechst 33342 (Beyotime, Shanghai, China, \#C1026) solution was added to coverslips for 10 minutes, then washed twice in PBS. Cell imaging was performed using an X51 fluorescence inverted microscope (Olympus, Japan).

\section{Western blot analysis}

Total proteins were extracted from heart tissues of TAC mice, cardiac fibroblasts, cardiomyocytes, and concentrated supernatants of the culture medium, and quantified using the BCA protein assay kit (Thermo Scientific, USA). According to the molecular weight of target proteins, the protein samples were separated using SDS/PAGE (10\% or $15 \%$ gel), then transferred to Immobilon-P PVDF membranes. After blocking with western blocking buffer (5\% BSA: bovine serum albumin $2.5 \mathrm{~g}+$ TBST $50 \mathrm{~mL}$ ), the membranes were incubated overnight at $4{ }^{\circ} \mathrm{C}$ with the following primary antibodies: CysC (1:1,000, ab109508, Abcam, Cambridge, MA, USA), phosphorylated extracellular-regulated protein kinase (pERK, 1:5,000, \#4370), tERK (1:5,000, \#4695), pP38 (1:1,000, \#4511), tP38 (1:1,000, \#8690), pJNK (1:1,000, \#4668), tJNK (1:1,000, \#9252), pTAK1 (1:1,000, \#4508), tTAK1 (1:1,000, \#5206), and GAPDH (1:10,000, \#8884, all Cell Signaling Technology, Danvers, MA, USA). Membranes were then incubated with horseradish peroxidase (HRP)-conjugated secondary antibodies $(1: 1,000)$ for 1 hour at room temperature. After treatment with ProLight chemiluminescent detection kit (Tiangen Biotech Inc., Beijing, China), the proteins were detected using Omega Lum C imaging system (Aplegen, CA, USA).

\section{Real-time PCR}

The gene expression levels of Cst3, Nppa, Nppb, and Myh7 were measured using real-time PCR (RT-PCR). Total RNA was extracted from cardiac fibroblasts or heart tissue using TRIzol reagent (Invitrogen, Carlsbad, CA, USA), and $1 \mu \mathrm{g}$ total RNA was reverse transcribed to form cDNA using the Toyobo RT-PCR kit. For relative quantification of RNA, SYBR Premix ExTaq kit (Cat\#: RR420A, Takara, Japan) was used for RT-PCR. The primers we used were synthesized by Sangon Biotech (Shanghai, China):
Cst3 (forward: TTCGCCGTAAGCGAGTACAACAA, reverse: CATTGGCATGGTCCTATGAGACT), Nppa (forward: TCGAGCAGATTTGGCTGTTATCTTC, reverse: TGACAGGATTGGAGCCCAGAG), Nppb (forward: TCCTTAATCTGTCGCCGCTG, reverse: GGCGCTGTCTTGAGACCTAA), Myh7 (forward: CCTAAGGTGCTGTTTCAAAGGC, reverse: AAGAGCCGTGACATTGGCG), GAPDH (forward TCCCTCAAGATTGTCAGCAA, reverse: AGATCCACAACGGATACATT). GAPDH was used as an internal control.

\section{Statistical analysis}

The count data is expressed as a percentage (\%), and the measurement data is expressed as mean \pm standard deviation. Count data was compared using chi-square test or Fisher's exact test, and measurement data was compared using independent sample $t$ test or analysis of variance (ANOVA). Cardiomyocyte CSA measurement and western blot grayscale analysis were performed using Image $\mathrm{J}$ software. Statistical graphs were drawn using GraphPad Prism 7 software (GraphPad Software Inc., San Diego, CA, USA). $\mathrm{P}<0.05$ was considered statistically significant. All analyses were carried out with SPSS 25.0 statistical package for Windows (SPSS Inc, Chicago, IL, USA).

\section{Results}

Serum CysC increased in patients with hypertension excluding the influence of renal function

A total of 1,045 patients were enrolled in this study. The baseline clinical characteristics of the study patients $(634$ with hypertension and 411 without hypertension) are illustrated in Table 1. There were no statistically significant differences in age, gender, history of myocardial infarction, chronic heart failure, diabetes, and chronic kidney disease, glycated hemoglobin, cTnT and NT-proBNP between the two groups. The levels of SCr were higher while eCCr was lower in patients with hypertension than those with no hypertension. In terms of echocardiographic parameters, after BSA correction, the LVMi and LADi of patients with hypertension were significantly higher than those without hypertension. There were more patients with E/A ratio $<1$ in the hypertensive group than in the non-hypertensive group. However, there were no significant differences between the two groups in cardiac hyperparameters such 
Table 1 Baseline clinical characteristics of the hypertension and non-hypertension groups

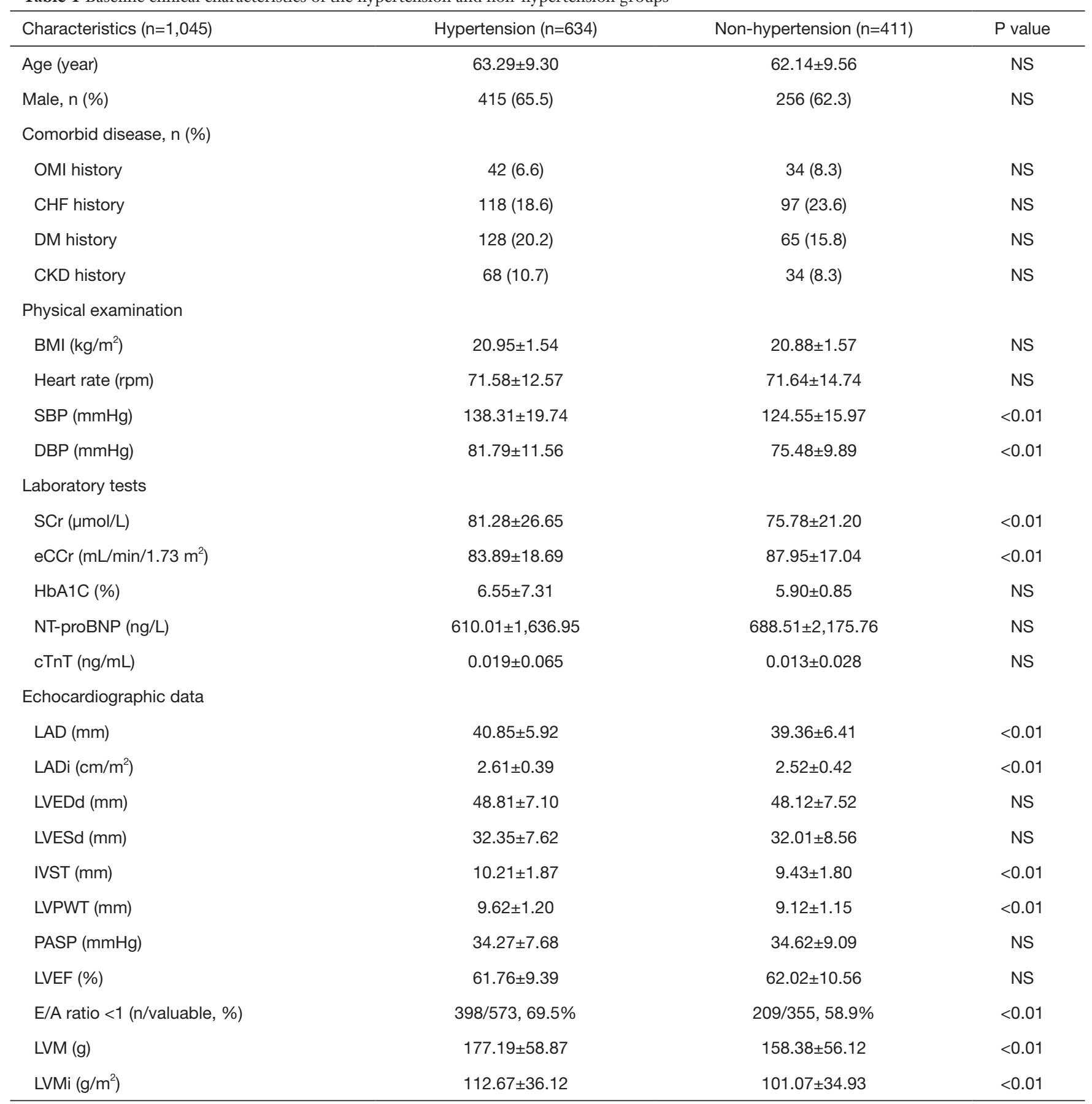

OMI, old myocardial infarction; CHF, congestive heart failure; DM, diabetes mellitus; CKD, chronic kidney disease; BMI, body mass index; SBP, systolic blood pressure; DBP, diastolic blood pressure; SCr, serum creatinine; eCCr, endogenous creatinine clearance rate; HbA1C, Glycosylated hemoglobin; NT-proBNP, N terminal pro B type natriuretic peptide; cTnT, Cardiac troponin T; LAD, left atrial diameter; LADi, left atrial diameter index; LVEDd, left ventricular end-diastolic diameter; LVESd, left ventricular end-systolic diameter; IVST, interventricular septal thickness; LVPWT, left ventricular posterior wall thickness; PASP, pulmonary artery systolic pressure; LVEF, left ventricular ejection fraction; LVM, left ventricular mass; LVMi, left ventricular mass index. 

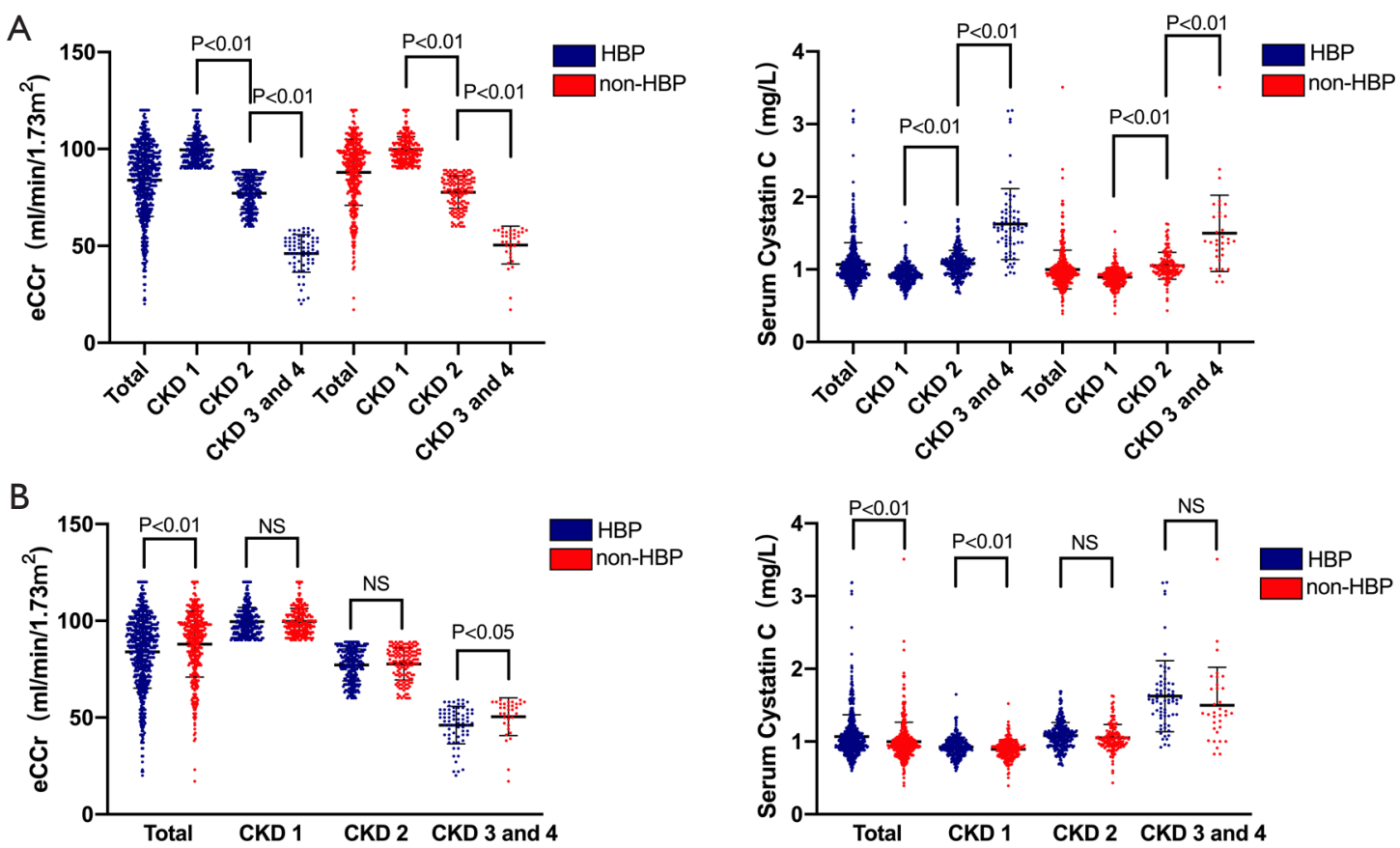

Figure 1 eCCr and serum CysC of total study patients, including patients with different renal functions classified by $\mathrm{CKD}$ staging. (A) With decreased renal function, the serum CysC increased across CKD stages in both the HBP and non-HBP groups; (B) across the total cases, serum CysC was higher in patients with hypertension $(1.07 \pm 0.30$ vs. $1.00 \pm 0.27 \mathrm{mg} / \mathrm{L}, \mathrm{P}<0.01)$ and eCCr was lower in patients with hypertension $\left(83.89 \pm 18.69\right.$ vs. $\left.87.95 \pm 17.04 \mathrm{~mL} / \mathrm{min} / 1.73 \mathrm{~m}^{2}, \mathrm{P}<0.01\right)$. In CKD stage 1 patients, serum CysC was higher $(0.93 \pm 0.13$ vs. $0.89 \pm 0.13 \mathrm{mg} / \mathrm{L}, \mathrm{P}<0.01)$ in patients with hypertension, while no statistically significant difference in eCCr was found between groups. In CKD stage 2-4 patients, there was no statistically significant difference in serum CysC between the two groups. CKD, chronic kidney disease; eCCr, endogenous creatinine clearance rate; HBP, hypertension; CysC, cystatin C.

as LVEDd, LVESd, PASP, and LVEF. Furthermore, the serum CysC levels were higher in patients with hypertension than those with no-hypertension $(1.07 \pm 0.30$ vs. $1.00 \pm 0.27 \mathrm{mg} / \mathrm{L}, \mathrm{P}<0.01$, Figure 1$)$.

Since serum CysC levels are mainly affected by renal function, we conducted a subgroup analysis based on patients' renal function. Baseline clinical characteristics of the study patients are illustrated in Table 2. In patients with CKD stage $1\left(\mathrm{eCCr} \geq 90 \mathrm{~mL} / \mathrm{min} / 1.73 \mathrm{~m}^{2}, 284\right.$ with hypertension and 233 without hypertension), there was no statistically significant difference in eCCr, NTproBNP, and LVEF between the hypertensive and the nonhypertensive group, while serum CysC increased $(0.93 \pm 0.13$ vs. $0.89 \pm 0.13 \mathrm{mg} / \mathrm{L}, \mathrm{P}<0.01)$ in the hypertensive group. Serum $\mathrm{Cys} C$ increased in patients with mildly impaired renal function in CKD stage $2\left(60 \mathrm{~mL} / \mathrm{min} / 1.73 \mathrm{~m}^{2}\right.$ $\leq \mathrm{eCCr}<90 \mathrm{~mL} / \mathrm{min} / 1.73 \mathrm{~m}^{2}, 282$ with hypertension and 144 without hypertension), though there was no statistical difference in eCCr and serum CysC between the two groups (CysC $1.08 \pm 0.18$ vs. $1.05 \pm 0.18 \mathrm{mg} / \mathrm{L}, \mathrm{P}=0.10)$. 404 Since there were fewer patients with CKD stage 3-4 405 $\left(15 \mathrm{~mL} / \mathrm{min} / 1.73 \mathrm{~m}^{2} \leq \mathrm{eCCr}<60 \mathrm{~mL} / \mathrm{min} / 1.73 \mathrm{~m}^{2}\right)$ among 406 the enrolled patients ( 68 with hypertension and 34 without hypertension), these patients were combined for analysis. The eCCr was lower in patients with hypertension $(46.09 \pm 9.69 \mathrm{vs}$. $50.41 \pm 9.78 \mathrm{~mL} / \mathrm{min} / 1.73 \mathrm{~m}^{2}, \mathrm{P}<0.05$ ) and the serum CysC of the two groups of patients were not statistically different (1.62 \pm 0.49 vs. $1.50 \pm 0.52 \mathrm{mg} / \mathrm{L}, \mathrm{P}=0.23$, Figure 1$)$.

\section{Correlation between serum CysC and $L V H$}

Linear regression analysis showed that serum $\mathrm{Cys} C$ was negatively correlated with eCCr $(\mathrm{r}=-0.724, \mathrm{P}<0.01)$ and LVEF $(r=-0.300, P<0.01)$, and was positively correlated with age $(r=0.311, \mathrm{P}<0.01)$, LVMi $(\mathrm{r}=0.296, \mathrm{P}<0.01)$, LADi $(\mathrm{r}=0.260, \mathrm{P}<0.01)$, cTnT $(\mathrm{r}=0.313, \mathrm{P}<0.01)$, and NTproBNP $(r=0.518, \mathrm{P}<0.01$, Figure 2$)$.

According to the level of LVMi, 1,045 patients were 
Table 2 Baseline clinical characteristics of patients with different renal function (CKD stage 1 and CKD stage 2)

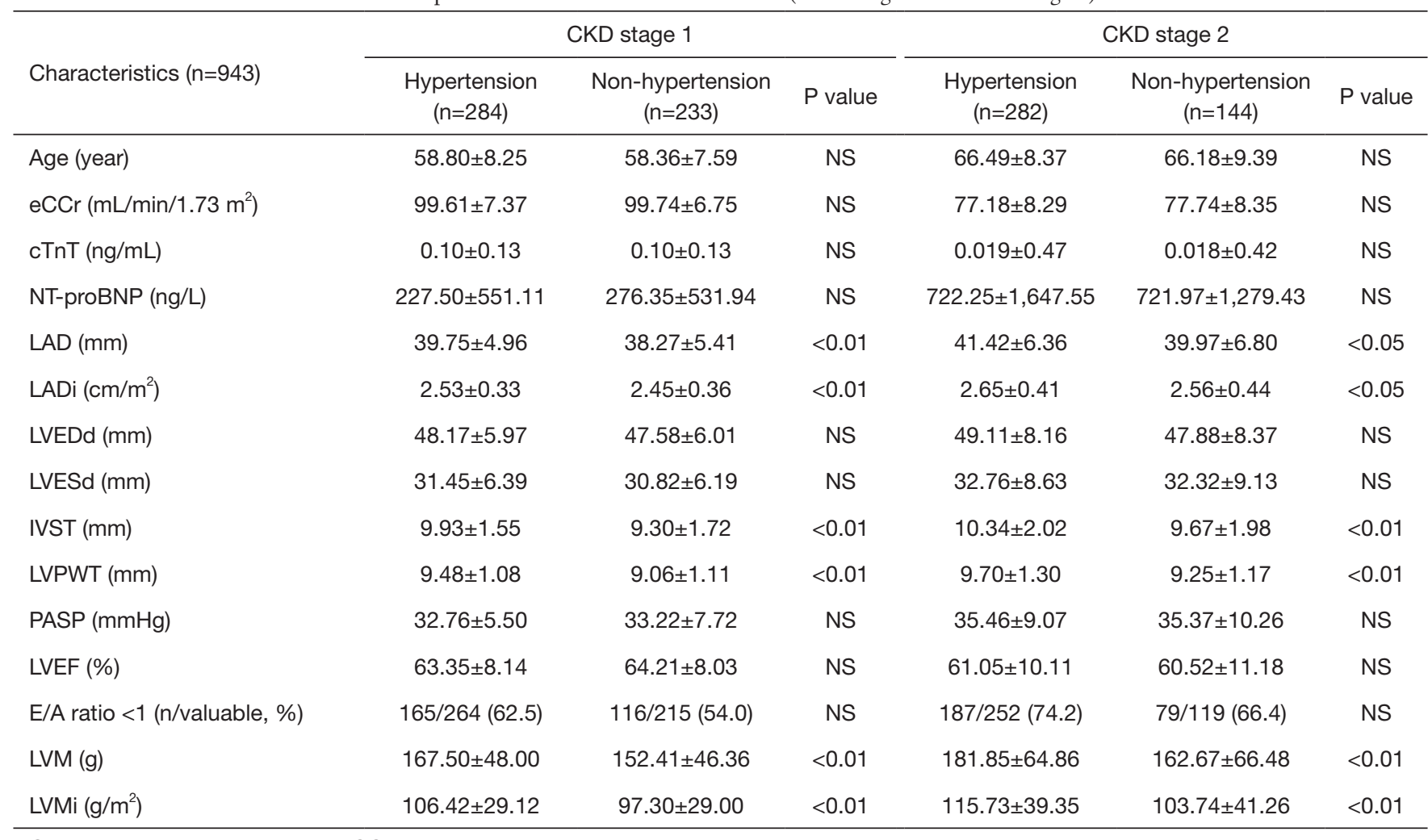

CKD, chronic kidney disease; eCCr, endogenous creatinine clearance rate; NT-proBNP, N terminal pro B type natriuretic peptide; cTnT, Cardiac troponin T; LAD, left atrial diameter; LADi, left atrial diameter index; LVEDd, left ventricular end-diastolic diameter; LVESd, left ventricular end-systolic diameter; IVST, interventricular septal thickness; LVPWT, left ventricular posterior wall thickness; PASP, pulmonary artery systolic pressure; LVEF, left ventricular ejection fraction; LVM, left ventricular mass; LVMi, left ventricular mass index.

divided into the LVH group (270 cases) and the non-LVH group (775 cases). The baseline clinical characteristics of the study patients are illustrated in Table 3. The baseline data showed that the LVH group had a higher proportion of males $(\mathrm{P}<0.05)$, and a higher proportion of patients with a history of hypertension, heart failure, and diabetes $(\mathrm{P}<0.01)$. The eCCr was lower in the $\mathrm{LVH}$ group $(\mathrm{P}<0.01)$. In terms of echocardiographic parameters, the LVH group showed increased LADi and LVMi $(\mathrm{P}<0.01)$, and decreased LVEF $(\mathrm{P}<0.01)$. Patients in the $\mathrm{LVH}$ group also had worse cardiac and renal functions, and serum $\mathrm{Cys} C$ levels were higher in the $\mathrm{LVH}$ group $(1.16 \pm 0.38$ vs. $1.00 \pm 0.23 \mathrm{mg} / \mathrm{L}, \mathrm{P}<0.01)$.

In order to exclude the effect of heart and renal dysfunction on serum CysC, a total of 493 hypertensive patients with CKD stage 1 and 2 without chronic heart failure were selected for a subgroup analysis (108 cases with LVH, 385 cases without LVH). There was no statistically significant difference in baseline data between the two groups in terms of gender, age, BMI, heart rate, history of old myocardial infarction (OMI) and diabetes. The SBP and NT-proBNP were higher in the $\mathrm{LVH}$ group $(\mathrm{P}<0.01)$. There was no statistically significant difference in eCCr between the two groups, while serum $\mathrm{CysC}$ levels were higher in the $\mathrm{LVH}$ group $(1.02 \pm 0.17$ vs. $0.98 \pm 0.16 \mathrm{mg} /$ $\mathrm{L}, \mathrm{P}<0.01)$. In terms of echocardiographic parameters, the LVH group showed increased LADi and LVMi $(\mathrm{P}<0.01)$ and decreased LVEF $(\mathrm{P}<0.05$, Figure 3). Further logistic regression analysis showed that after correction for LVEF, eCCr, and NT-proBNP, increased SBP $(\mathrm{P}=0.047)$, increased CysC $(\mathrm{P}=0.029)$, and increased LADi $(\mathrm{P}<0.01)$ were independent risk factors for $\mathrm{LVH}$ in hypertensive patients with CKD stage 1 and 2 without cardiac dysfunction.

\section{CysC content increased in the myocardium of TAC mice}

The wild-type C57BL/6 mice showed a significant increase 

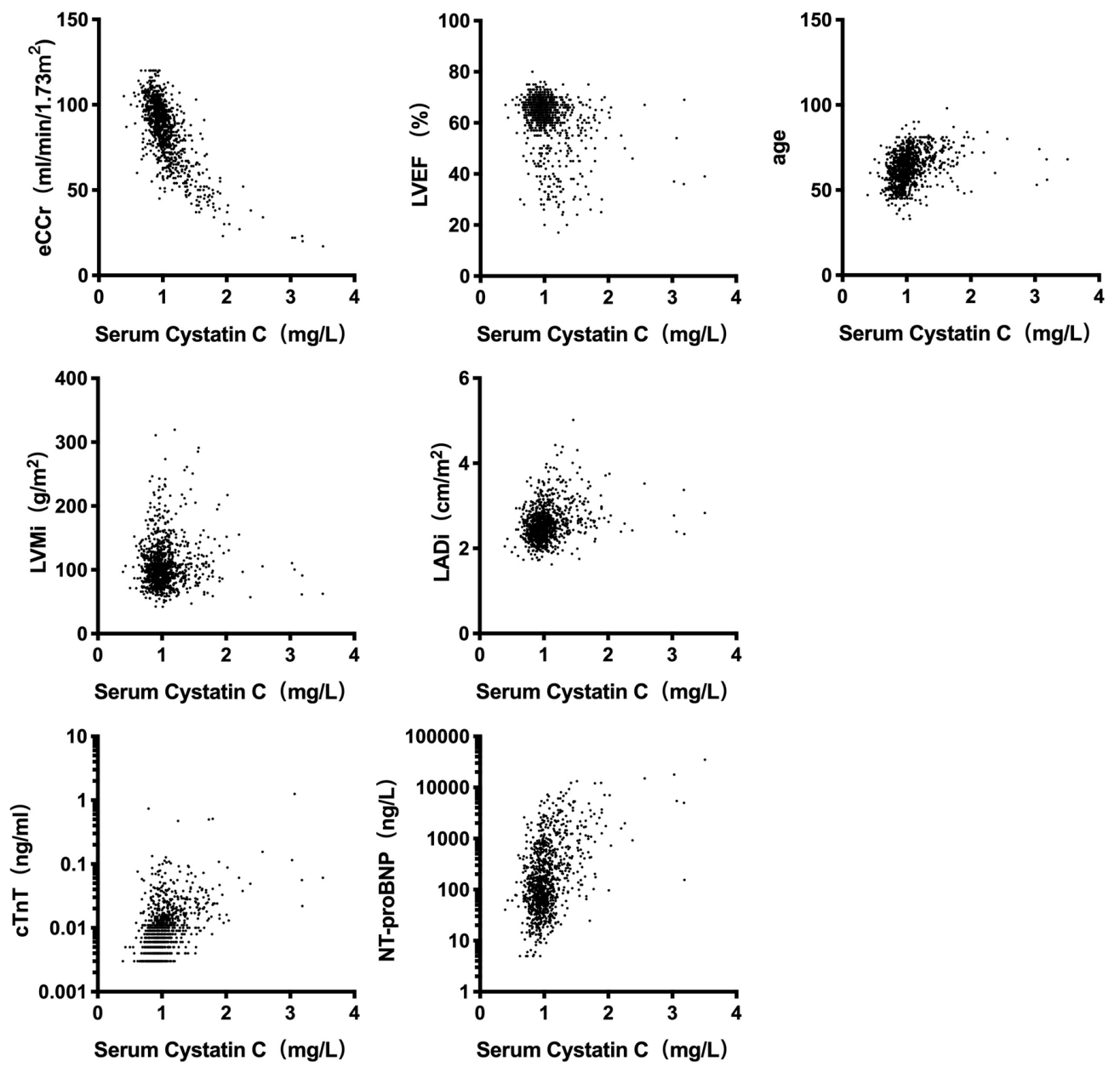

Figure 2 Correlation between serum $\mathrm{Cys} C$ and age, laboratory, and echocardiographic parameters. Linear regression analysis showed that serum CysC was negatively correlated with eCCr $(\mathrm{r}=-0.724, \mathrm{P}<0.01)$ and LVEF $(\mathrm{r}=-0.300, \mathrm{P}<0.01)$, but was positively correlated with age ( $\mathrm{r}=0.311, \mathrm{P}<0.01)$, LVMi (r=0.296, $\mathrm{P}<0.01)$, LADi ( $\mathrm{r}=0.260, \mathrm{P}<0.01)$, cTnT $(\mathrm{r}=0.313, \mathrm{P}<0.01)$, and NT-proBNP ( $=0.518, \mathrm{P}<0.01)$. eCCr, endogenous creatinine clearance rate; LVEF, left ventricular ejection fraction; LVMi, left ventricular mass index; LADi, left atrial diameter index; NT-proBNP, N terminal pro B type natriuretic peptide; cTnT, Cardiac troponin T; CysC, cystatin C.

in the volume of cardiac specimens at 14 and 28 days after TAC. Echocardiography showed myocardial hypertrophy in TAC mice at day 14, and a significant enlargement of the LV in TAC mice at day 28. The hemodynamic parameters suggested that LVESP and LVEDP were higher on day 14 and day 28 after TAC compared to the sham-operated group, indicating successful model induction. C57BL/6 wild type mice were found to have increased CysC levels in cardiac tissue 3 days after TAC, which increased further at 7 days after TAC, then decreased to baseline levels at 14 days after TAC. After 28 days, the CysC levels increased again, but there was no significant difference (Figure 4).
Expression and secretion of CysC increased in primary rat 474 cardiomyocytes after mechanical stretch

The neonatal rat cardiomyocytes were cultured on a silicone sheet pre-coated with rat tail collagen in $0.1 \%$ acetic acid. After 48 hours of culture, cardiomyocytes fused together and were beating with a pulsation frequency of approximately $100-120$ beats/min. After replacement with serum-free F12/DMEM medium for 12 hours, cells were given mechanical stretch stimulation.

The CST3 gene expression level of cardiomyocytes increased significantly after mechanical stretch stimulation. The CysC levels of the primary cardiomyocytes increased 
Table 3 Baseline clinical characteristics of the LVH and non-LVH groups

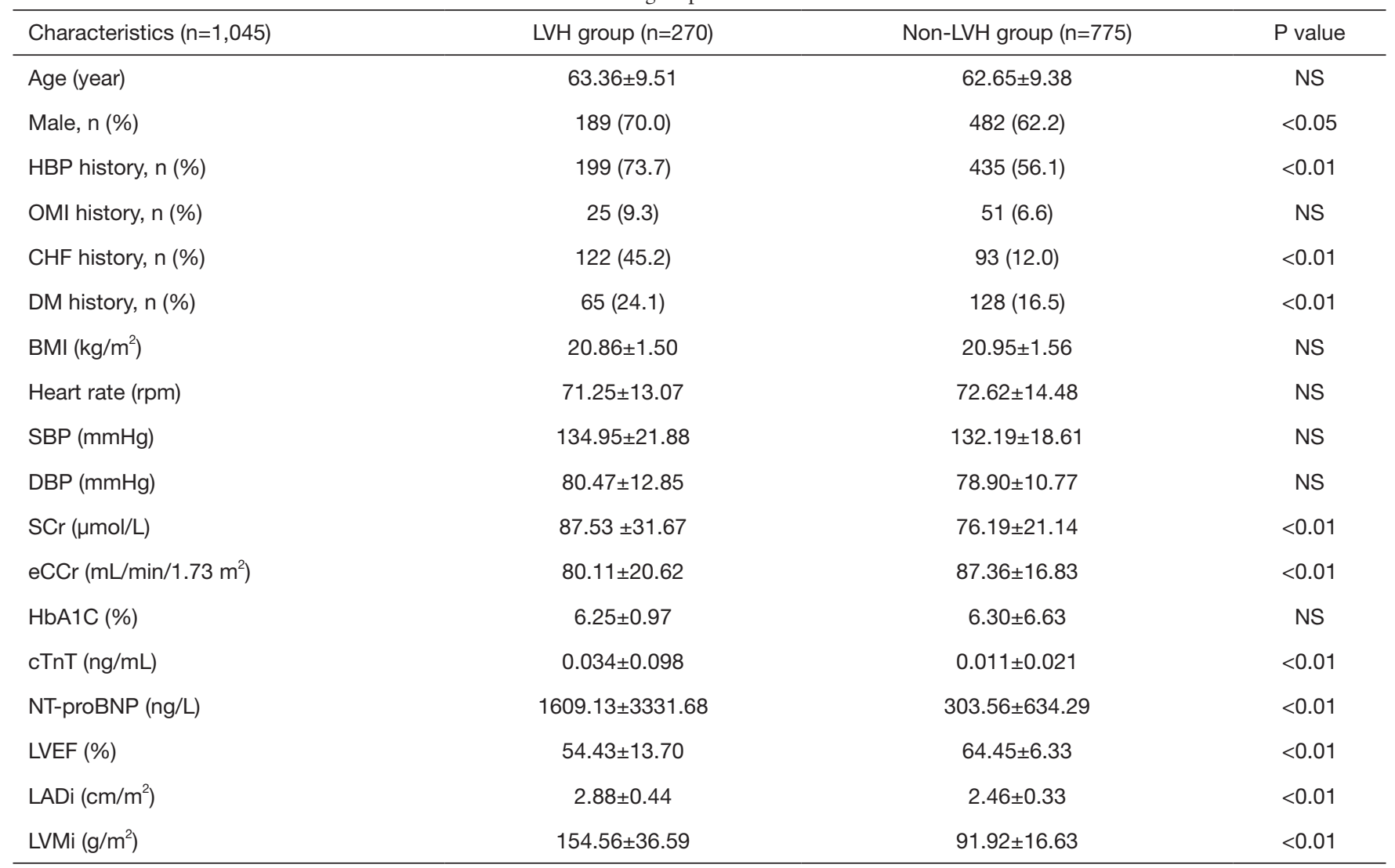

LVH, left ventricular hypertrophy; HBP, hypertension; OMI, old myocardial infarction; CHF, congestive heart failure; DM, diabetes mellitus; BMI, body mass index; SBP, systolic blood pressure; DBP, diastolic blood pressure; SCr, serum creatinine; eCCr, endogenous creatinine clearance rate; HbA1C, Glycosylated hemoglobin; NT-proBNP, N terminal pro B type natriuretic peptide; cTnT, Cardiac troponin T; LVEF, left ventricular ejection fraction; LADi, left atrial diameter index; LVMi, left ventricular mass index.

significantly 15 minutes after mechanical stretch stimulation, and showed a continuous increase until 24 hours after stimulation. CysC levels began to increase about 3 hours after mechanical stretch stimulation in the supernatant of cells.

Furthermore, the CST3 gene expression levels of cardiac fibroblasts also increased after stimulation. The intracellular CysC levels of the cardiac fibroblasts increased significantly 15 minutes after mechanical stretch then returned to baseline levels at 6 and 12 hours after intervention. However, the level of secreted CysC in the supernatants did not increase. Instead, the concentration decreased at 3 and 6 hours after intervention (Figure 5).

\section{The bypertrophic effect of CysC on rat primary cardiomyocytes}

Rat primary cardiomyocytes were divided into a control group and an exogenous CysC $(500 \mathrm{ng} / \mathrm{mL})$ group. After 24 hours of intervention, $\alpha$-actinin immunofluorescence staining was used to label cardiomyocytes. It was found that the CSA of cardiomyocytes in the CysC intervention group was larger than the control group $(2,135 \pm 70.53 \mathrm{vs}$. $1,267 \pm 59.15 \mu^{2}, \mathrm{P}<0.01$, Figure 6).

After administration of CysC $125 \mathrm{ng} / \mathrm{mL}$ for 6 hours, the expression of $\mathrm{Nppb}$ increased in the rat primary cardiomyocytes, but the expression of $\mathrm{Nppa}$ and Myh7 did not increase significantly. After administration of CysC $500 \mathrm{ng} / \mathrm{mL}$ for 6 hours, the expression of $\mathrm{Nppa}, \mathrm{Nppb}$, and Myh7 all increased significantly.

After intervention with CysC $500 \mathrm{ng} / \mathrm{mL}$ in rat primary cardiomyocytes, ERK phosphorylation increased after 10 minutes, then gradually returned to baseline levels. Given different concentrations of CysC, ERK phosphorylation at 10 minutes also increased gradually with the increase of $\mathrm{Cys} \mathrm{C}$ concentration, showing a dose-dependent response. 

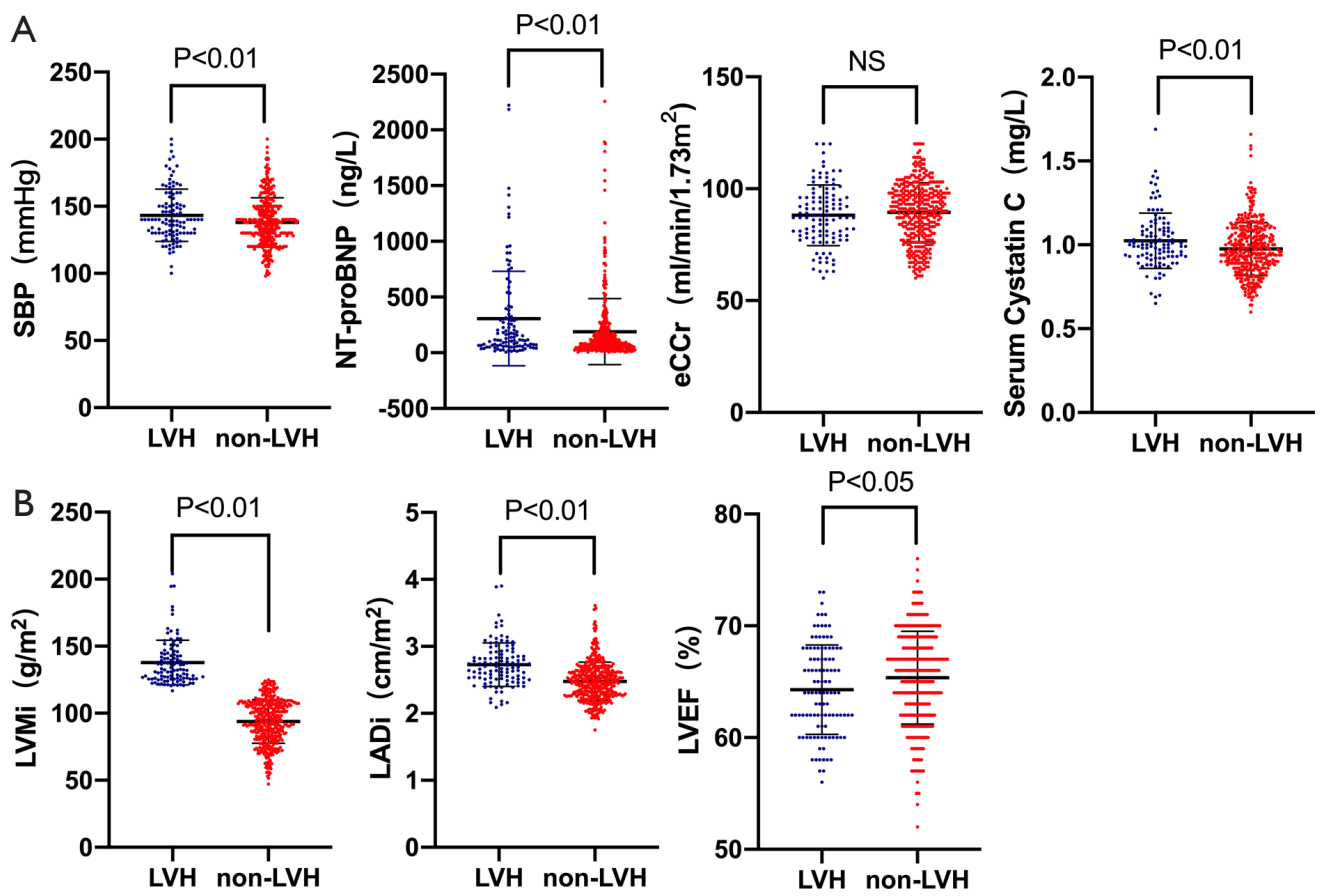

Figure 3 Difference between LVH and non-LVH groups in hypertensive patients with CKD stage 1 and 2 without heart failure. SBP and NT-proBNP were higher in the LVH group $(\mathrm{P}<0.01)$. There was no statistically significant difference in eCCr between the two groups, while serum CysC was higher in the $\mathrm{LVH}$ group $(1.02 \pm 0.17$ vs. $0.98 \pm 0.16 \mathrm{mg} / \mathrm{L}, \mathrm{P}<0.01)$. In terms of echocardiographic parameters, the LVH group showed increased LADi and LVMi $(\mathrm{P}<0.01)$ and decreased LVEF $(\mathrm{P}<0.05)$. SBP, systolic blood pressure; NT-proBNP, N terminal pro B type natriuretic peptide; eCCr, endogenous creatinine clearance rate; LVMi, left ventricular mass index; LADi, left atrial diameter index; LVEF, left ventricular ejection fraction; LVH, left ventricular hypertrophy; CKD, chronic kidney disease; CysC, cystatin C.

This suggests that $\mathrm{Cys} C$ has a dose-dependent hypertrophic effect on rat primary cardiomyocytes. In terms of other MAPKs, p38 phosphorylation increased 1 hour after intervention with CysC, while JNK phosphorylation did not increase. Furthermore, given different concentrations of $\mathrm{CysC}$, TAK1 phosphorylation at 10 minutes after CysC intervention also increased gradually with the increase of CysC concentration (Figure 6).

Western blot showed that CysC protein synthesis in cells and secretion in supernatants of primary cardiomyocytes increased at 1 and 3 hours after AngII stimulation. However, the levels of $\mathrm{Cys} C$ in cells and secretory CysC in the supernatants were significantly decreased after the interference of CST3-siRNA, even when stimulated with AngII. The CST3-siRNA interference reduced CysC protein synthesis in cardiomyocytes as well as secretory CysC in the culture supernatant.

It was evident that after exogenous $\mathrm{Cys} C$ intervention, the levels of $\mathrm{Cys} C$ protein in cardiomyocytes increased significantly. CST3-siRNA was transfected to inhibit the expression of $\mathrm{Cys} C$ in cardiomyocytes, and $\mathrm{Cys} C$ content in both the cardiomyocytes and in supernatants were significantly decreased. With the subsequent administration of exogenous CysC, the levels of $\mathrm{Cys} C$ in cardiomyocytes increased significantly, and gradually increased with time, while the content of $\mathrm{Cys} C$ protein in the supernatants of the medium gradually decreased. This suggests that exogenous CysC can enter into cardiomyocytes (Figure 7).

\section{Discussion}

\section{Serum CysC in patients with bypertension}

In this study, all the enrolled patients were divided into a hypertension group or a non-hypertension group. There was no significant difference in NT-proBNP and LVEF between the two groups, while the LVMi and LADi were significantly higher in patients with hypertension. Overall, this is consistent with previous studies, where patients with hypertension have lower eCCr levels and higher serum $\mathrm{Cys} C$ levels. This is because hypertensive patients often 
A

Sham

TAC 14d
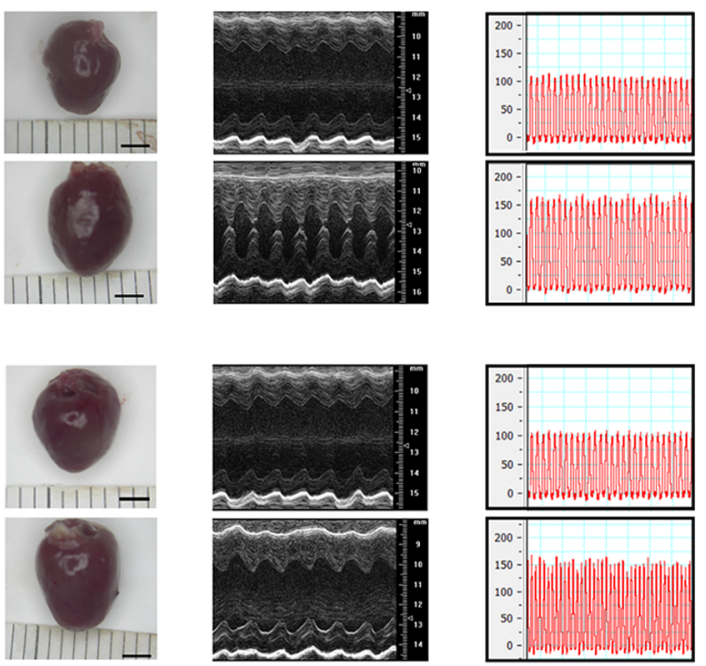

Echocardiogram

of left ventricular

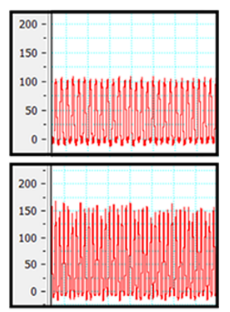

Left ventricular

pressure
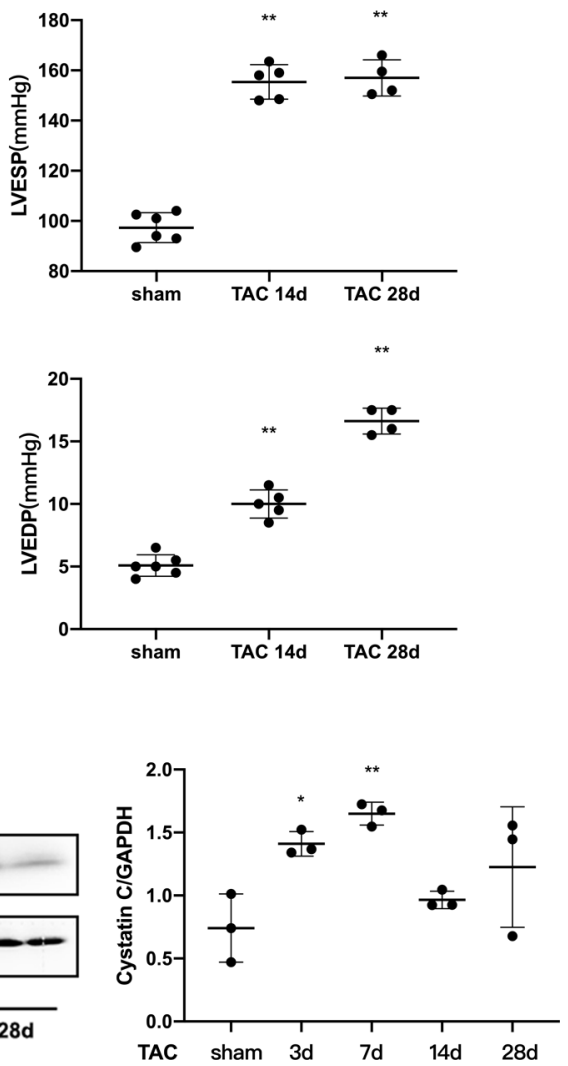

Figure 4 Changes in CysC levels in the myocardium of TAC mice. (A) C57BL/6 wild type mouse heart echocardiogram and left ventricular pressure (LVESP and LVEDP) 14 and 28 days after TAC; (B) CysC levels in TAC mouse myocardium (*, P<0.05; **, P<0.01). TAC, transverse aortic constriction; LVEDP, left ventricular end-diastolic pressure; LVESP, left ventricular end-systolic pressure; GAPDH, glyceraldehyde-3-phosphate dehydrogenase.

have concomitant kidney damage, and CysC can effectively reflect a decline in renal filtration function (7).

In order to rule out the influence of renal function, we performed a subgroup analysis according to renal function. It was found that in patients with CKD stage 1 (eCCr $\left.\geq 90 \mathrm{~mL} / \mathrm{min} / 1.73 \mathrm{~m}^{2}\right)$, there was no statistically significant difference in eCCr between the hypertensive group and the non-hypertensive group, but the serum $\mathrm{Cys} C$ levels in the hypertensive group were significantly increased. However, the serum $\mathrm{Cys} C$ of hypertensive patients was still higher than that of patients without hypertension, and the difference was not related to a decrease in renal function. In other words, hypertension itself may cause an increase in serum $\mathrm{Cys} C$ through certain underlying mechanisms. The fluctuation of serum CysC levels in hypertensive patients may also reflect the pressure overload on the heart.

In this study, we saw that in patients with CKD stage 2, there was no statistically significant difference in the eCCr and serum CysC between the hypertensive group and the non-hypertensive group, suggesting that in people with mild renal impairment, serum CysC levels were affected mainly by renal function, masking the effect of CysC secreted by the heart itself.

\section{Relationship between serum CysC and pathological changes in cardiac structure}

The changes in cardiac structure that can occur in patients with hypertension before heart failure include an enlarged left atrium, LVH, and left ventricular diastolic dysfunction. Among them, in the early stages of hypertension, LVH mainly manifests as centripetal hypertrophy, predominantly due to pressure overload (27).

Hypertension with structural changes in the heart is an important factor contributing to poor prognosis. In previous studies, comparing patients with CysC $\geq 1.28 \mathrm{mg} / \mathrm{L}$ 


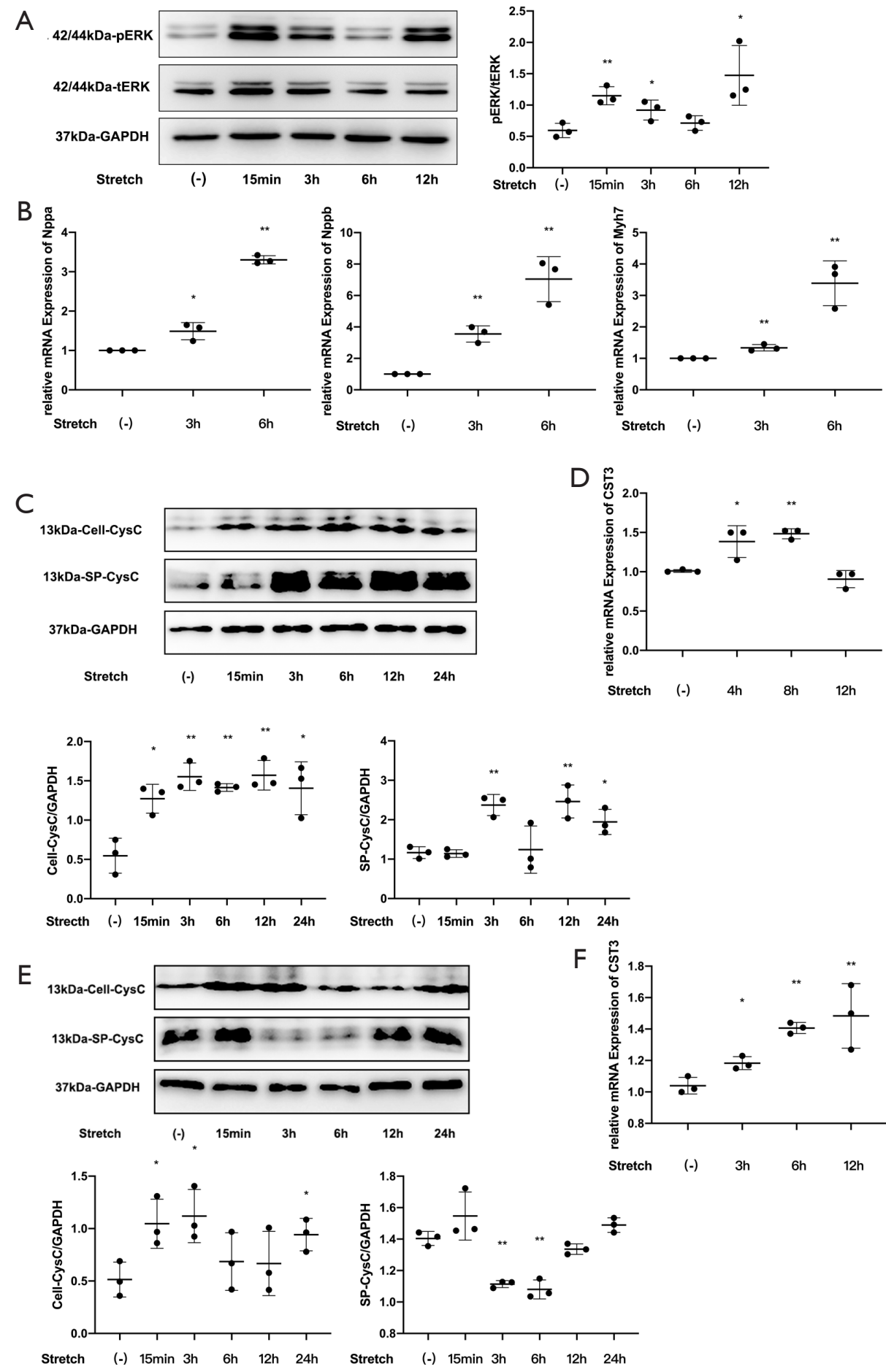

Figure 5 Changes in the expression and secretion of $\mathrm{Cys} C$ after mechanical stretch stimulation of rat primary cardiomyocytes and cardiac fibroblasts. (A) ERK phosphorylation after mechanical stretch of primary cardiomyocytes; (B) gene expression of Nppa, Nppb, and Myh7 of primary cardiomyocytes after mechanical stretch; (C) intracellular CysC (Cell-CysC) and secreted CysC in the culture supernatant (SP-CysC) after mechanical stretch of primary cardiomyocytes; (D) expression level of CST3 gene after mechanical stretch of primary cardiomyocytes; (E) intracellular CysC and secreted CysC in the culture supernatant after mechanical stretch of fibroblasts; (F) expression level of CST3 gene after mechanical stretch of fibroblasts $\left({ }^{*}, \mathrm{P}<0.05 ;{ }^{* *}, \mathrm{P}<0.01\right)$. ERK, extracellular regulated protein kinase; GAPDH, glyceraldehyde-3phosphate dehydrogenase; CysC, cystatin C; SP, supernatant. 


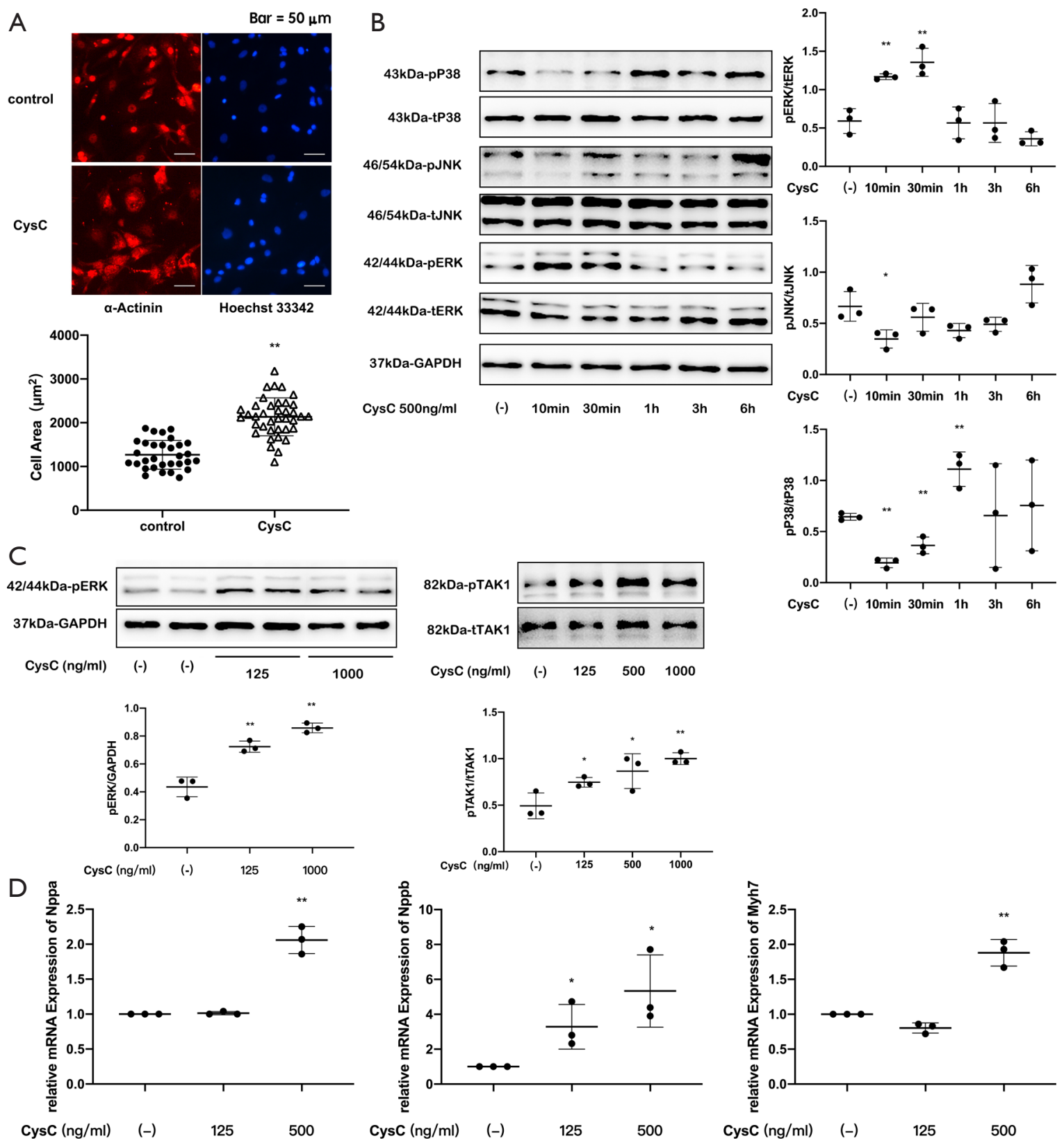

Figure 6 Effects of $\mathrm{Cys} C$ on the development of myocardial hypertrophy in rat primary cardiomyocytes. (A) Immunofluorescence staining of $\alpha$-actinin in primary cardiomyocytes and measurement of cross-sectional area; (B) MAPK phosphorylation of primary cardiomyocytes after intervention with CysC $(500 \mathrm{ng} / \mathrm{mL})$ at different time points; (C) ERK and TAK1 phosphorylation of primary cardiomyocytes after 10-minute intervention with CysC of different concentrations; (D) gene expression of Nppa, Nppb, and Myh7 of primary cardiomyocytes after 6-hour intervention with $\mathrm{CysC}$ of different concentrations ${ }^{*}, \mathrm{P}<0.05$; $\left.{ }^{* *}, \mathrm{P}<0.01\right)$. ERK, extracellular regulated protein kinase; JNK, c-Jun N-terminal kinase; TAK1, transforming growth factor activated kinase-1; GAPDH, glyceraldehyde-3-phosphate dehydrogenase; CysC, cystatin C. 
A

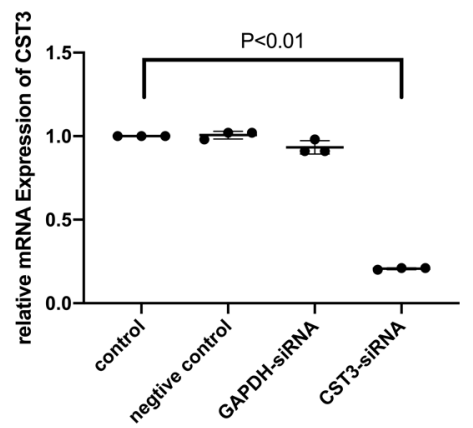

B

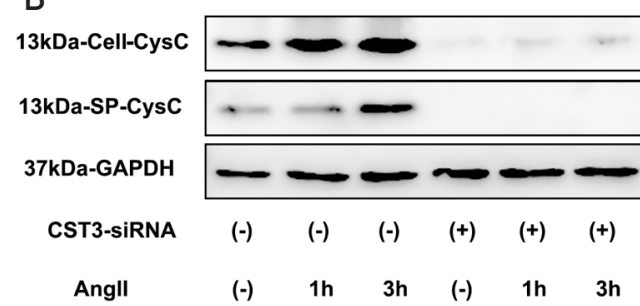

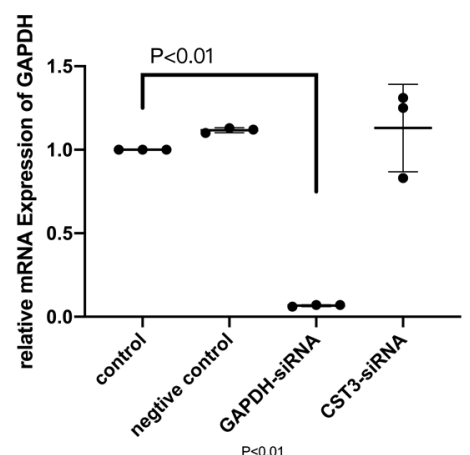

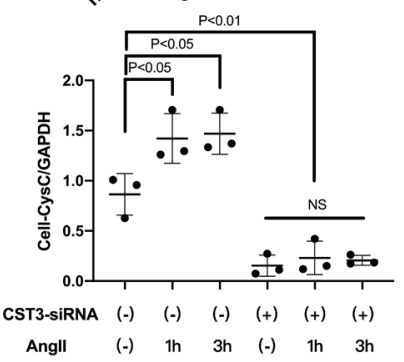

C

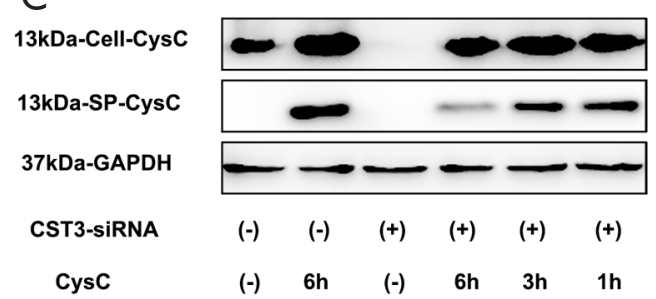

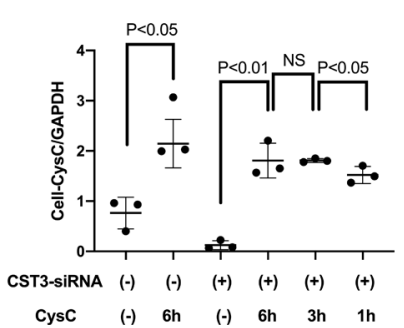
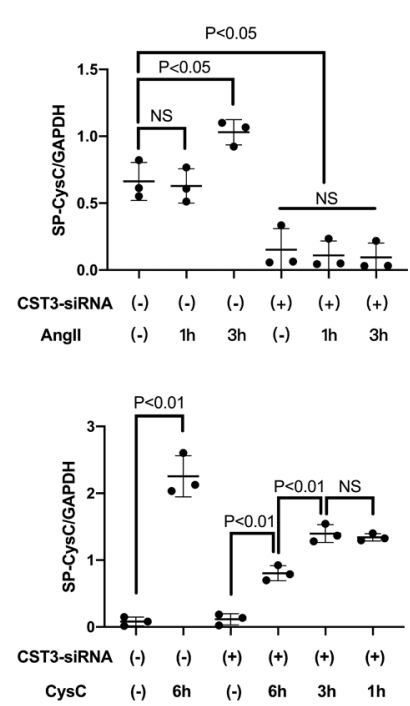

Figure 7 Levels of $\mathrm{Cys} C$ protein in cardiomyocytes and in supernatants after exogenous $\mathrm{Cys} C$ intervention. (A) Gene expression of CST3 and GAPDH in primary cardiomyocytes after 24-hour interference of CST3-siRNA or GAPDH-siRNA; (B) CysC content in primary cardiomyocytes (cell-CysC) and in supernatants (SP-CysC) after 24-hour interference of CST3-siRNA, followed by AngII $10^{-6}$ $\mathrm{M}$ intervention; (C) CysC levels in primary cardiomyocytes and in supernatants after 24-hour interference of CST3-siRNA, followed by exogenous CysC intervention. AngII, Angiotensin II; GAPDH, glyceraldehyde-3-phosphate dehydrogenase; CysC, cystatin C; SP, supernatant.

and $\mathrm{CysC} \leq 0.91 \mathrm{mg} / \mathrm{L}$, the incidence of $\mathrm{LVH}$ was $68 \%$ and $44 \%$ with an odds ratio 2.17 (95\% confidence interval 1.34 to $3.52, \mathrm{P}=0.002$ ), while the incidence of left ventricular diastolic dysfunction was $52 \%$ and $24 \%$, with an odds ratio 1.79 (95\% confidence interval $1.04-3.11$, $\mathrm{P}=0.04)$ (34). The Dallas Heart study also suggested that the increase in serum CysC is related to $\mathrm{LVH}$ and the increase in left ventricular mass as shown by magnetic resonance imaging (10). Furthermore, with the increase of serum CysC, the incidence of eccentric and centripetal myocardial hypertrophy increased $(\mathrm{P}=0.0008)$. In particular, the incidence of concentric myocardial hypertrophy significantly increased $(\mathrm{P}=0.0187)$, and was independent of age, gender, history of hypertension, eGFR based on creatinine, medication use, and other factors (35).

In this study, it was found that LVH group had worse renal and cardiac functions. In the subgroup analysis of hypertensive patients, elevated serum $\mathrm{Cys} C$ was an independent risk factor for $\mathrm{LVH}$ in hypertensive patients with normal or mildly reduced renal function without chronic heart failure, which is consistent with previous research. After excluding the interference of other confounding factors, a direct correlation between serum CysC and $\mathrm{LVH}$ was still observed.

Elevated serum $\mathrm{Cys} C$ is related to the occurrence of hypertension and myocardial hypertrophy, and the possible reasons include: Firstly, chronic renal insufficiency and LVH have similar pathophysiological mechanisms, 
often accompanied by aging, hypertension, and diabetes. Secondly, chronic renal insufficiency itself can also affect cardiac structure and function changes. Sakuragi et al. found that $55 \%$ of patients with CKD stage $2-3$ have LVH (35). The accumulation of uremic toxins during renal insufficiency can even occur within the normal range of eGFR, and participate in the occurrence of $\mathrm{LVH}$ and interstitial fibrosis $(36,37)$. Thirdly, we speculate that the slight increase in serum $\mathrm{Cys} C$ in hypertensive patients may come from the secretion of the heart. Early in the course of hypertension, due to the increase in arterial blood pressure, the heart may be able to secrete more CysC. The heartderived CysC secreted to the outside of the cell may even directly be involved in the regulation of the pathogenesis of cardiac hypertrophy caused by hypertension.

It should be noted that $\mathrm{Cys} C$ can be secreted by almost all the nucleated cells throughout the body, and the local secretion of $\mathrm{Cys} C$ in the heart has little effect on the absolute value of serum $\mathrm{CysC}$. Hence, increased serum $\mathrm{Cys} C$ is mainly affected by decreases in renal function. When the heart is subjected to increased pressure load, CysC secretion may increase, but it is unlikely to significantly affect the $\mathrm{Cys} C$ in circulation. Therefore, it is difficult to find a cut-off value of serum $\mathrm{Cys} C$ for clinical diagnosis. Nevertheless, it is still meaningful to explore the role and mechanism of $\mathrm{CysC}$ in hypertension-induced myocardial hypertrophy. In the case of relatively stable circulating CysC levels, local CysC in the myocardium may also participate in the occurrence of myocardial hypertrophy through paracrine effects, forming a positive feedback or negative feedback mechanism. Exploring its mechanism may help discover new therapeutic targets.

\section{Effect of pressure overload on the expression and secretion of cardiac CysC}

Our laboratory previously performed iTRAQ analysis of cultured medium from cardiomyocytes or cardiac fibroblasts subjected to mechanical stretch for 24 hours, compared to control. We found that myocardial cell CysC protein secretion increased in the case of mechanical stretch, however, fibroblast $\mathrm{Cys} C$ protein secretion decreased after mechanical stretch.

We constructed a mouse TAC model to simulate the increase in pressure on the heart. Since the concentration of serum CysC is mainly affected by renal function, it is difficult to evaluate the changes of cardiac expression and secretion of $\mathrm{Cys} C$ in TAC mice according to the serum
Cys $\mathrm{C}$ content. Therefore, we measured the levels of 676 $\mathrm{Cys} C$ protein in the myocardial tissue of TAC mice. TAC 677 mice developed myocardial hypertrophy approximately 678 2 weeks after modeling, and heart failure occurred around 679 4 weeks. The content of $\mathrm{CysC}$ in myocardial tissue 680 increased significantly 3 days after TAC and reached a 681 peak on day 7 , which was significantly earlier than the 682 occurrence of myocardial hypertrophy that was seen 683 by echocardiography. Although previous literature has 684 shown that the rate of serum CysC production is relatively 685 stable, and its serum concentration is mainly affected by 686 glomerular filtration rate, in our study, we found that in 687 the TAC mouse model the heart expressed CysC protein 688 under pressure overload. However, we also found that the 689 expression of $\mathrm{Cys} C$ in cardiac tissue increased in TAC 690 mice in the early postoperative period as well as 4 weeks 691 after TAC, and the expression of $\mathrm{CysC}$ dropped slightly 692 2 weeks after TAC. This may be due to the gradual progress 693 of myocardial hypertrophy around 2 weeks after TAC with 694 the accumulation of myocardial interstitium, in which 695 a large amount of CysC is secreted and participates in 696 pathophysiological changes.

We established a mechanical stretch model of primary cardiomyocytes and fibroblasts to simulate the situation of pressure overload in vitro. This is also consistent with our clinical data and the conclusions obtained from TAC mice suggesting that the expression and secretion of CysC increases when the heart is subjected to pressure overload. In addition, we also found that the increased secretion of CysC protein mainly comes from cardiomyocytes rather than fibroblasts, and the expression of $\mathrm{CysC}$ increases almost immediately after pressure overload. The secreted CysC in the supernatant also increases, which is consistent with the results of our iTRAQ analysis.

Previous studies have shown that the synthesis, secretion, and serum concentration of $\mathrm{Cys} C$ are strictly regulated by various factors, such as thyroid function, glucocorticoids, C-reactive protein levels, smoking, pregnancy status $(38,39)$, cancer, HIV infection, cardiovascular disease, and nervous system diseases (40-42). In this study, in addition to finding that mechanical stretch can induce increased expression and secretion of $\mathrm{CysC}$ in cardiomyocytes, we also explored the effects of AngII stimulation on primary cardiomyocytes. It was found that $\mathrm{Cys} C$ protein content in cardiomyocytes and secreted $\mathrm{CysC}$ increased 1 hour and 3 hours after AngII stimulation. Previous studies have confirmed that TGF $\beta 1$ levels are significantly increased during mechanical stretch and AngII stimulation, and plays a synergistic role (43-46).

\section{7}

698

699

700

701 
Studies have also found that TGF $\beta 1$ can upregulate $\mathrm{Cys} C$ secretion of vascular smooth muscle cells, mouse embryonic cells, cultured differentiated podocytes, 3T3-L1 fibroblasts, and human lung fibroblasts $(42,47,48)$. Therefore, we speculate that the mechanism of $\mathrm{Cys} C$ expression and secretion induced by mechanical stretch/Ang II may be related to the secretion of cytokines such as TGF $\beta 1$.

\section{Effect of increased extracellular CysC on cardiomyocytes}

The pathogenesis of myocardial hypertrophy caused by hypertension includes the combined action of cardiomyocytes and fibroblasts. In the case of pressure overload on the heart, early hypertrophy of cardiomyocytes leads to compensatory myocardial hypertrophy. Myocardial hypertrophy is the result of the combined effects of neurohumors, cytokines, and other factors. Among them, increased mechanical load is the most important cause, and MAPK signaling pathways including ERK, p38, and JNK are the most important signal pathway mediating cardiac hypertrophy.

Previous studies have shown that $\mathrm{Cys} C$ can promote the adhesion of neonatal rat cardiomyocytes. It can increase the adherence of cardiomyocytes by $67 \%$ within 8 hours and can increase DNA synthesis by $76 \%$ after 24 hours. It also synergizes with growth factors such as insulin and epidermal growth factor (49). It is suggested that $\mathrm{CysC}$ can promote the growth of primary cardiomyocytes.

In this study, in order to simulate the increase of secreted $\mathrm{CysC}$, we administered exogenous CysC. The selected $\mathrm{Cys} C$ intervention concentration was consistent with the physiological concentration of rat $\mathrm{Cys} C$ reported in the literature. It was observed that after the addition of exogenous $\mathrm{Cys} C$ in the primary cardiomyocyte culture medium, $\alpha$-actinin immunofluorescence stained cardiomyocyte actin showed an increase in cardiomyocyte volume, RT-PCR showed that Nppa, Nppb, and Myh7 were upregulated, and ERK phosphorylation levels increased significantly. This suggests that CysC has a hypertrophic effect on cardiomyocytes.

We also performed further experiments to figure out the mechanism of the hypertrophic effect of CysC on cardiomyocytes. We demonstrated in this study that after exogenous CysC intervention, the levels of extracellular CysC gradually decreased with time-the $\mathrm{CysC}$ in the supernatant of the culture medium decreased to about $50 \%$ at 6 hours after the intervention, and the levels of $\mathrm{CysC}$ in the cells gradually increased. In addition to the increase in ERK phosphorylation levels, TAK1 and p38 772 phosphorylation levels also increased significantly after 773 exogenous $\mathrm{Cys} C$ intervention. Therefore, we speculate that 774 exogenous CysC enters cardiomyocytes through certain 775 mechanisms, and meanwhile mediate the hypertrophy 776 of cardiomyocytes through direct regulation of signaling $\quad 777$ pathways.

The phenomenon that extracellular secretory CysC can enter cells via internalization has been reported in other cell lines (50-52). Internalization of extracellular secretory CysC was observed in human cell lines, and flow cytometry and confocal microscopy showed that during CysC incubation, intracellular CysC increased and remained at least 6 hours after 5 minutes, reaching 4-6 times baseline levels. Western blot showed that the internalized $\mathrm{Cys} C$ was not degraded and was fully functional (51). Immunofluorescence staining can observe the obvious presence of $\mathrm{Cys} C$ stained vesicles in the cells (52). The uptake of extracellular CysC in the proximal tubules directly binds to the endocytic receptor megalin in the proximal tubule cells in a calciumdependent manner and enters the proximal tubule cells via endocytosis (50). There is as yet no research confirming the mechanism by which CysC enters into cardiomyocytes.

In our study, it was found that ERK, TAK1, and p38 phosphorylation levels in cardiomyocytes were significantly higher than the control group after exogenous CysC intervention. Our currently ongoing experiments show that in the 293 $\mathrm{T}$ cell line transfected with AT1R receptor then given exogenous CysC stimulation, ERK and TAK1 phosphorylation levels did not increase. This suggests the MAPKs were not activated by AT1R in exogenous CysC intervention. During hypertrophy of cardiomyocytes, the non-classical TGF- $\beta$ signaling pathway binds the extracellular TGF- $\beta$ molecule to the TGF- $\beta$ type II receptor, activating the key protein-TGF- $\beta$ activated kinase (TAK1), and further downstream MAPK signaling pathways, such as ERK, p38, and JNK $(53,54)$. In a study of malignant tumors, Sokol et al. found that CysC can block the binding of TGF $\beta$ and TGF $\beta$ type II receptor through interaction with this receptor, and inhibit the activation of the TGF- $\beta$ signaling pathway by TGF $\beta(47,55)$. Therefore, CysC has a structural basis for binding to TGF $\beta$ type II receptors. Furthermore, it has been reported that the TGF $\beta$ receptor can mediate ligand endocytosis. Liu et al. (56) reported that in zebrafish, the actin-binding protein Fscn1 can specifically interact with TGF $\beta$ type I receptors, and promote TGF $\beta$ type I. The TGF $\beta$ type II receptor complex internalizes and forms clathrin-coated vesicles, thereby 
promoting the transport of internalized receptors.

Therefore, we speculate that $\mathrm{CysC}$ may also interact with TGF $\beta$ II receptors which may then be endocytosed into cardiomyocytes, meanwhile regulating the downstream MAPK signaling pathways directly and mediating the hypertrophy of cardiomyocytes. We are now performing further experiments such as co-immunoprecipitation and laser confocal microscopy to clarify the direct role of $\mathrm{CysC}$ and TGF $\beta$ type II receptors in cardiac hypertrophy.

\section{Acknowledgments}

Funding: This work was supported by National Key Research and Development Program of China (2018YFC1312703) and National Natural Science Foundation of China (81730009, 81941002, 81700312).

\section{Footnote}

Reporting Checklist: The authors have completed the ARRIVE reporting checklist. Available at http://dx.doi. org/10.21037/atm-20-7041

Data Sharing Statement: Available at http://dx.doi. org/10.21037/atm-20-7041

Conflicts of Interest: All authors have completed the ICMJE uniform disclosure form (available at http://dx.doi. org/10.21037/atm-20-7041). The authors have no conflicts of interest to declare.

Ethical Statement: The authors are accountable for all aspects of the work in ensuring that questions related to the accuracy or integrity of any part of the work are appropriately investigated and resolved. All procedures performed in studies involving human participants were in accordance with the Helsinki Declaration (as revised in 2013). The study was approved by the local Ethics Committee. All participants provided written informed consent to clinical examinations, laboratory analyses, and the use of data records for research purposes. All animal experiments were approved by the Animal Care and Use Committee of Fudan University and in compliance with the Guidelines for the Care and Use of Laboratory Animals published by the National Academies Press (NIH publication number: 85-23, revised 1996).

Open Access Statement: This is an Open Access article distributed in accordance with the Creative Commons Attribution-NonCommercial-NoDerivs 4.0 International License (CC BY-NC-ND 4.0), which permits the noncommercial replication and distribution of the article with the strict proviso that no changes or edits are made and the original work is properly cited (including links to both the formal publication through the relevant DOI and the license). See: https://creativecommons.org/licenses/by-nc-nd/4.0/.

\section{References}

1. Williams B, Mancia G, Spiering W, et al. 2018 ESC/ESH Guidelines for the management of arterial hypertension. Eur Heart J 2018;39:3021-104.

2. Wachter R, Halbach M, Bakris GL, et al. An exploratory propensity score matched comparison of second-generation and first-generation baroreflex activation therapy systems. J Am Soc Hypertens 2017;11:81-91.

3. Bohm M, Mahfoud F, Ukena C, et al. First report of the Global SYMPLICITY Registry on the effect of renal artery denervation in patients with uncontrolled hypertension. Hypertension 2015;65:766-74.

4. Lobo MD, Sobotka PA, Stanton A, et al. Central arteriovenous anastomosis for the treatment of patients with uncontrolled hypertension (the ROX CONTROL HTN study): a randomised controlled trial. Lancet 2015;385:1634-41.

5. Chow SL, Maisel AS, Anand I, et al. Role of Biomarkers for the Prevention, Assessment, and Management of Heart Failure: A Scientific Statement From the American Heart Association. Circulation 2017;135.

6. Newman DJ, Thakkar H, Edwards RG, et al. Serum cystatin $\mathrm{C}$ measured by automated immunoassay: A more sensitive marker of changes in GFR than serum creatinine. Kidney Int 1995;47:312-8.

7. Pavkov ME, Knowler WC, Hanson RL, et al. Comparison of serum cystatin C, serum creatinine, measured GFR, and estimated GFR to assess the risk of kidney failure in American Indians with diabetic nephropathy. Am J Kidney Dis 2013;62:33-41.

8. Gao P, Zhao K, Wang X-M, et al. Association Between Serum Cystatin C and High Blood Pressure (HBP): A Cross-Sectional Study of an Elder Chinese Type 2 Diabetic Population. Clin Lab 2015;61:1401-7.

9. Otsuka T, Kato K, Kachi Y, et al. Serum cystatin C, creatinine-based estimated glomerular filtration rate, and the risk of incident hypertension in middle-aged men. Am J Hypertens 2014;27:596-602. 
10. Patel PC, Ayers CR, Murphy SA, et al. Association of cystatin $\mathrm{C}$ with left ventricular structure and function: the Dallas Heart Study. Circulation: Heart Failure 2009;2:98-104.

11. Androulakis E, Papageorgiou N, Lioudaki E, et al. Subclinical Organ Damage in White-Coat Hypertension: The Possible Role of Cystatin C. J Clin Hypertens (Greenwich) 2017;19:190-7.

12. Tousoulis D, Androulakis E, Papageorgiou N, et al. Genetic predisposition to left ventricular hypertrophy and the potential involvement of cystatin-C in untreated hypertension. Am J Hypertens 2013;26:683-90.

13. Huerta A, López B, Ravassa S, et al. Association of cystatin $\mathrm{C}$ with heart failure with preserved ejection fraction in elderly hypertensive patients: potential role of altered collagen metabolism. J Hypertens 2016;34:130-8.

14. Breidthardt T, Sabti Z, Ziller R, et al. Diagnostic and prognostic value of cystatin $\mathrm{C}$ in acute heart failure. Clin Biochem 2017;50:1007-13.

15. Waheed S, Matsushita K, Astor BC, et al. Combined association of creatinine, albuminuria, and cystatin $\mathrm{C}$ with all-cause mortality and cardiovascular and kidney outcomes. Clin J Am Soc Nephrol 2013;8:434-42.

16. Salgado JV, França AK, Cabral NA, et al. Cystatin C, kidney function, and cardiovascular risk factors in primary hypertension. Rev Assoc Med Bras (1992) 2013;59:21-7.

17. Shlipak MG, Matsushita K, Ärnlöv J, et al. Cystatin C versus creatinine in determining risk based on kidney function. N Engl J Med 2013;369:932-43.

18. Shlipak MG, Katz R, Sarnak MJ, et al. Cystatin C and prognosis for cardiovascular and kidney outcomes in elderly persons without chronic kidney disease. Ann Intern Med 2006;145:237-46.

19. van der Laan SW, Fall T, Soumaré A, et al. Cystatin C and Cardiovascular Disease: A Mendelian Randomization Study. J Am Coll Cardiol 2016;68:934-45.

20. Sai E, Shimada K, Miyauchi K, et al. Increased cystatin C levels as a risk factor of cardiovascular events in patients with preserved estimated glomerular filtration rate after elective percutaneous coronary intervention with drugeluting stents. Heart Vessels 2016;31:694-701.

21. Svensson-Färbom $P$, Ohlson Andersson M, Almgren P, et al. Cystatin $\mathrm{C}$ identifies cardiovascular risk better than creatinine-based estimates of glomerular filtration in middle-aged individuals without a history of cardiovascular disease. J Intern Med 2014;275:506-21.

22. Chung ES, Packer M, Lo KH, et al. Randomized, doubleblind, placebo-controlled, pilot trial of infliximab, a chimeric monoclonal antibody to tumor necrosis factoralpha, in patients with moderate-to-severe heart failure:

964 results of the anti-TNF Therapy Against Congestive Heart Failure (ATTACH) trial. Circulation 2003;107:3133-40.

23. Yamazaki T, Komuro I, Yazaki Y. Role of the reninangiotensin system in cardiac hypertrophy. Am J Cardiol 1999;83:53H-7H.

24. Mancia G, Fagard R, Narkiewicz K, et al. 2013 ESH/ESC guidelines for the management of arterial hypertension: the Task Force for the Management of Arterial Hypertension of the European Society of Hypertension (ESH) and of the European Society of Cardiology (ESC). Eur Heart J 2013;34:2159-219.

25. KDIGO. KDIGO 2012 Clinical Practice Guideline for the Evaluation and Management of Chronic Kidney Disease. Kidney Int Suppl 2012;3:1-150.

26. Devereux RB, Alonso DR, Lutas EM, et al. Echocardiographic assessment of left ventricular hypertrophy: comparison to necropsy findings. Am J Cardiol 1986;57:450-8.

27. Ganau A, Devereux RB, Roman MJ, et al. Patterns of left ventricular hypertrophy and geometric remodeling in essential hypertension. J Am Coll Cardiol 1992;19:1550-8.

28. Ponikowski P, Voors AA, Anker SD, et al. 2016 ESC Guidelines for the diagnosis and treatment of acute and chronic heart failure. Eur J Heart Fail 2016;18:891-975.

29. Zou Y, Liang Y, Gong H, et al. Ryanodine receptor type 2 is required for the development of pressure overload-induced cardiac hypertrophy. Hypertension 2011;58:1099-110.

30. Wu J, Bu L, Gong H, et al. Effects of heart rate and anesthetic timing on high-resolution echocardiographic assessment under isoflurane anesthesia in mice. J Ultrasound Med 2010;29:1771-8.

31. Ma H, Gong H, Chen Z, et al. Association of Stat3 with HSF1 plays a critical role in G-CSF-induced cardioprotection against ischemia/reperfusion injury. J Mol Cell Cardiol 2012;52:1282-90.

32. Chen Z, Xu J, Ye Y, et al. Urotensin II inhibited the proliferation of cardiac side population cells in mice during pressure overload by JNK-LRP6 signalling. J Cell Mol Med 2014;18:852-62.

33. Jiang G, Gong H, Niu Y, et al. Identification of Amino Acid Residues in Angiotensin II Type 1 Receptor Sensing Mechanical Stretch and Function in Cardiomyocyte Hypertrophy. Cell Physiol Biochem 2015;37:105-16.

34. Ix JH, Shlipak MG, Chertow GM, et al. Cystatin C, left ventricular hypertrophy, and diastolic dysfunction: data from the Heart and Soul Study. J Card Fail 2006;12:601-7. 
35. Sakuragi S, Ichikawa K, Yamada K, et al. Serum cystatin $\mathrm{C}$ level is associated with left atrial enlargement, left ventricular hypertrophy and impaired left ventricular relaxation in patients with stage 2 or 3 chronic kidney disease. Int J Cardiol 2015;190:287-92.

36. Barreto FC, Barreto DV, Liabeuf S, et al. Serum Indoxyl Sulfate Is Associated with Vascular Disease and Mortality in Chronic Kidney Disease Patients. Clin J Am Soc Nephrol 2009;4:1551-8.

37. Lekawanvijit S, Adrahtas A, Kelly DJ, et al. Does indoxyl sulfate, a uraemic toxin, have direct effects on cardiac fibroblasts and myocytes? Eur Heart J 2010;31:1771-9.

38. Knight EL, Verhave JC, Spiegelman D, et al. Factors influencing serum cystatin $\mathrm{C}$ levels other than renal function and the impact on renal function measurement. Kidney Int 2004;65:1416-21.

39. Risch L, Herklotz R, Blumberg A, et al. Effects of glucocorticoid immunosuppression on serum cystatin $\mathrm{C}$ concentrations in renal transplant patients. Clin Chem 2001;47:2055-9.

40. Kos J, Stabuc B, Cimerman N, et al. Serum cystatin C, a new marker of glomerular filtration rate, is increased during malignant progression. Clin Chem 1998;44:2556-7.

41. Harman AN, Kraus M, Bye CR, et al. HIV-1-infected dendritic cells show 2 phases of gene expression changes, with lysosomal enzyme activity decreased during the second phase. Blood 2009;114:85-94.

42. Shi GP, Sukhova GK, Grubb A, et al. Cystatin C deficiency in human atherosclerosis and aortic aneurysms. J Clin Invest 1999;104:1191-7.

43. Li JM, Brooks G. Differential protein expression and subcellular distribution of TGF beta(1), beta(2), and beta(3) in cardiomyocytes during pressure overload induced hypertrophy. J Mol Cell Cardiol 1997;29:2213-24.

44. Yu CM, Tipoe GL, Lai KWH, et al. Effects of combination of angiotensin-converting enzyme inhibitor and angiotensin receptor antagonist on inflammatory cellular infiltration and myocardial interstitial fibrosis after acute myocardial infarction. J Am Coll Cardiol 2001;38:1207-15.

45. Sun YO, Zhang JQ, Zhang JK, et al. Angiotensin II, transforming growth factor-beta(1) and repair in the infarcted heart. J Mol Cell Cardiol 1998;30:1559-69.

46. Hao JM, Wang BQ, Jones SC, et al. Interaction between angiotensin II and Smad proteins in fibroblasts in failing heart and in vitro. Am J Physiol Heart Circ Physiol
2000;279:H3020-H30.

47. Sokol JP, Schiemann WP. Cystatin C antagonizes transforming growth factor signaling in normal and cancer cells. Mol Cancer Res 2004;2:183-95.

48. Kasabova M, Joulin-Giet A, Lecaille F, et al. Regulation of TGF-beta 1-driven Differentiation of Human Lung Fibroblasts: emerging roles of cathepsin B and cystatin C. J Biol Chem 2014;289:16239-51.

49. Sun Q, Chen G, Feng J. Sulfhydryl protease inhibitory peptide promotes the adhesion and growth of neonatal rat cardiomyocytes. Journal of Beijing Medical University $1993 ; 25: 281-3$.

50. Kaseda R, Iino N, Hosojima M, et al. Megalin-mediated endocytosis of cystatin $\mathrm{C}$ in proximal tubule cells. Biochem Biophys Res Commun 2007;357:1130-4.

51. Ekstrom U, Wallin H, Lorenzo J, et al. Internalization of cystatin C in human cell lines. FEBS J 2008;275:4571-82.

52. Wallin H, Bjarnadottir M, Vogel LK, et al. Cystatins - Extra- and intracellular cysteine protease inhibitors: High-level secretion and uptake of cystatin $\mathrm{C}$ in human neuroblastoma cells. Biochimie 2010;92:1625-34.

53. Watkins SJ, Jonker L, Arthur HM. A direct interaction between TGFbeta activated kinase 1 and the TGFbeta type II receptor: implications for TGFbeta signalling and cardiac hypertrophy. Cardiovasc Res 2006;69:432-9.

54. Watkins SJ, Borthwick GM, Oakenfull R, et al. Angiotensin II-induced cardiomyocyte hypertrophy in vitro is TAK1-dependent and Smad2/3-independent. Hypertens Res 2012;35:393-8.

55. Sokol JP, Neil JR, Schiemann BJ, et al. The use of cystatin $\mathrm{C}$ to inhibit epithelial-mesenchymal transition and morphological transformation stimulated by transforming growth factor-beta. Breast Cancer Res 2005;7:R844-53.

56. Liu Z, Ning G, Xu R, et al. Fscn1 is required for the trafficking of TGF-beta family type I receptors during endoderm formation. Nat Commun 2016;7:12603.

(English Language Editor: C. Betlazar-Maseh)

Cite this article as: Shen Y, Zhang X, Li C, Wang X, Ye Y, Yuan J, Gong H, Zou Y, Ge J. Pressure overload promotes cystatin $\mathrm{C}$ secretion of cardiomyocytes to regulate the MAPK signaling pathway and mediate cardiac hypertrophy. Ann Transl Med 2020;8(22):1514. doi: 10.21037/atm-20-7041 